\title{
Combined Effect of Biofertilizers and Putrescine Amine on Certain Physiological Aspects and Productivity of Date Palm (Phoenix dactylifera L.) Grown in Reclaimed-Saline Soil
}

\author{
M. N. Helaly ${ }^{*}$ and Hanan A. R. E-Hosieny ${ }^{* *}$ \\ *Agriculture Botany Department, Faculty of Agriculture, Mansoura \\ University and ${ }^{* *}$ Horticulture Research Institute, Agricultural \\ Research Centre, Cairo, Egypt.
}

\begin{abstract}
A MINES play an important role in the plant response to adverse environmental conditions including salt and osmotic stress. In this investigation, the complemented effect of putrescine amine (put), biofertilizers and mycorhiza on date palm zaghloul genotype irrigated with salinized water and grown in reclaimed salinized soil was studied. The data indicated that the selected plant growth promoting rhizobacteria in the presence of putrescine amine make and/or enable date palm zaghloul genoty pe to increase its tolerance and adaptation to grow in the reclaimed-salinized soil. The treatments reduced saltinduced oxidative damage, increased productivity of date palm and improved its fruit quality. These results may be due to the increase in the photosynthetic pigments, organic solutes, promoting growth substances (GA, IAA and cytokinins), and activities of oxidant enzymes. Moreover, a decrease in the levels of lipid peroxidation and inhibitor substances (ABA) may be related. The most effective interaction treatments were pronounced at $2.5 \mathrm{mM}$ putresine due to more increase in the activities of ascorbate peroxidase (APOX) glutathione reductase (GR) and superoxide dismutase (SOD) and more decrease in lipid peroxidation. The interaction treatments with put at 5 $\mathrm{mM}$ increased disamine oxidase (DAO) and poly amine oxidase (PAO) activities compared to the other interaction treatments. The activates of these two enzy mes produce hy drogen peroxide $\mathrm{H}_{2} \mathrm{O}_{2}$ which may act in structural defense as a signal molecule and decreasing the production of polyamines against salt-induced oxidative damage in date palm. Further investigations are needed to explain the mechanisms develop in date palm grown in stress condition.
\end{abstract}

Keywords: Biofertilizer, Mycorhiza, Rhizobacteria, Putrescine (put), Date palm (Phoenix dactylifera L.), reclaimed saline soil.

Date palm (Phoenix dactylifera L.) the dioecious, monocotyledon species belonging to the family Arecaceae is a multipurpose tree having food, medicinal and ornamental importance. With the present uncertainty in the world food supply and the expected increase in demand, the date palm could be a good source of food of high nutritional value (Anonymous.., 2010). From the economical standpoint, date palm soft cultivars grown in Egypt differ in their sensitivity to salinity (El-Khawaga, 2013). Furthermore, growers have 
mistakenly believed that date palm does not require much attention, while the successful orchard management practices are the way to high yield of good fruit quality. One of the best tools of horticultural practices is fertilization. The use of fertilizers to increase yield is an important factor in all agricultural systems (Dong et al., 2005). Salt stress like other environmental stresses, induces adverse effects on growth, development, survival and productivity of date palm plants like other plant species by producing oxidative damage generation of reactive oxygen species (ROS), (Minura et al., 2003). It imposes both an ionic and an osmotic stress (Borsani et al., 2001) and becoming ever more prevalent as the intensity of agriculture increments (Zhu, 2002). It is developmentally regulated and stage specific phenomenon in many plant species (Tang and Newton, 2005). Plant cell utilizes three different strategies for coping with ionic and osmotic stress. 1) Osmotic adjustment of the cytoplasm due to the accumulations of compatible solutes such as betaine and proline (Tang and Newton, 2005). 2) Salt extrusion from the cell across the plasma membrane using ion transporters such as the $\mathrm{Na}^{+} / \mathrm{H}^{+}$antiporter (Minura et al., 2003) and 3) Salt accumulation in vacuoles using tonoplast transporters (Zhu 2005). Helaly and Hanan El-Hosieny (2011) showed that stress conditions increased lipid peroxidation or induce oxidative (stress) in plants tis sues as a high by degree of membrane deterioration. They added that lipid peroxidation can be initiated by ROS which severely affects functionality and integrity of cell membranes. It requires active $\mathrm{O}_{2}^{-}$uptake and involves the production of superoxide radical $\left(\mathrm{O}_{2}^{-}\right)$. The other highly reactive chemical species involving singlet oxygen $\left(\mathrm{O}_{2}^{-}\right)$hydroxyl free radical $\left(\mathrm{OH}^{-}\right)$and $\mathrm{H}_{2} \mathrm{O}_{2}$ all of which initiate lipid peroxidation (Dhindsa et al., 1981). Hence, constitutive and/or induced activity of SOD and other antioxidants such as POX, APOX, CAT and GR is essential. According to Seckin et al (2010) MDA has been frequently described as a suitable biomarker for lipid peroxidation under stress condition. biofertilizers and polyamines (putrescine and others) have been reported to be involved in the plant response to salt and osmotic stress by playing an important role in the ROS mediated damage caused by salt-stress (Zhu 2002 and Rasmia \& Darwesh 2013). Moreover, they behave as antioxidants in the protective mechanisms (Tang \& Newton, 2005 and Salama et al., 2014).

On the other hand, it was found that biological fertilizers play key role in productivity and sustainability of soil and also protect the environment as ecofriendly and cost effective inputs for the farmers (Mohammadi and Sohrabi, 2012). They added that with using the biological and organic fertilizers, a low input system can be carried out and it can help achieving sustainability of farms. Effective Microorganism humic acid and compost enriched with actinomyces agents were very effective in alleviating the adverse effect of salinity on date palm (El-Khawaga, 2013). Similary, the application of microorganisms at the rate of $90 \mathrm{ml} / \mathrm{palm} /$ one year combined with potassium sulphate at $1.5 \mathrm{~kg} / \mathrm{palm} /$ year as a soil application enhanced leaf chlorophyll content, fruit set percentage, retained fruit percentage, yield, fruit quality and leaf minerals content of "Hayany" date palm cv. (Salama et al., 2014). 
In the most plant species, salinity modify functional polyamine production and its distribution within the plant organs (Ghoulan et al., 2001) represented by a decrease in putrescine (Put) and increases in spermidine (Spd) and/or spermine (Spm). Depletion of endogenous polyamine levels due to salinity was previously reported (Groppa et al., 2003). In addition, putrescine (Put) is a low molecular weight polyamine compound which has been successfully applied to induce salt tolerance in many plant species (Borsani et al., 2001) by enhancing ROS generation during photosynthesis and plant developmental processes. Tang and Newton (2005) reported that putrescine was accumulated to high levels in the roots of the salt-resistant Virginia pine plants compared with the salt-sensitive plants, while an opposite trend was recorded in the shoots. They added that put increased the activities of key enzymes involved in oxidative stress such as ascorbate peroxidase (APOX), glutathione reductase (GR) and superoxide dismutase (SOD) and decreased lipid peroxidation. However, no clear relationship was observed between the mean level of salt resistance and the endogenous levels of putrescine. Moreover, the mechanism of tolerance at specific stage of plant development is a common approache and needed to introduce genetic and/or environmental and physiological improvement to salt stress tolerance.

In order to elucidate the role of biofertilizers and putrescine in reducing salt stress induced oxidation, the present study was undertaken to investigate the combined effect of plant growth promoting rhizobacteria (mixture of Azospirillum lipoferum, Paenibacillus polymyxa and Bacillus circulans), mycorhiza (AM) and dressing application with putrascine (put) at $0,2.5,5, \mathrm{mM}$ individually or incombination on certain physiological aspects and productivity quality of date palm (Phoenix dactylifera L.) zaghloul cv. irrigated with salinized water and grown in reclaimed-salinized soil.

\section{Material and Methods}

Two field experiments were carried out in reclaimed-salinized soil at the Agriculture Experimental Station located at Kalabshow and Zayan, Faculty of Agric, Mansoura Univ. Egypt during the two growing seasons of 2013 and 2014. The mechanical and chemical analyses of the experimental soil as well as the chemical analysis of the irrigation water used were estimated (Jackson, 1973 and Black et al., 1982) and presented in Table 1.

\section{Micro organisms inoculants}

Azospirillum lipoferum was grown on semi-solid $\mathrm{N}$-free malate medium (Doberiner, 1978) whereas Paenibacillus polymyxa and Bacillus circulans were individually grown on nutrient broth media (Dowson, 1957). They were separately suspended into sterile water and incubated after 5 days for 2-3 days at $30^{\circ} \mathrm{C}$ to maintain population of $3 \times 10^{8}$ colony forming unit $\mathrm{m}^{-1}(\mathrm{CFU} / \mathrm{ml})$. The mixed inoculums of the microbial cells were prepared by mixing equal volumes of the desired cell suspensions. All microbial strains were kindly provided from 
the Biofertilizers unit Fac. of Agric. Ain shams Univ. Egypt. Arbuscular mycorhiza fungus (Glomus mosseae) was grown on pot cultures containing onion plants for 4 months and the mycorhiza inoculums used were consisted of roots, hyphae, spores and growth media of the pot cultures. The standard inoculums $\left(400 \mathrm{~kg} / 4200 \mathrm{~m}^{2}\right)$ contained about $270 \mathrm{spors} / \mathrm{g}$. They were obtained from Agric. Microbial Dept. Soil, Water and Environment Res. Ins (SWER), Agric. Res. Center (ARC) Egypt. Fungus spores were measured by a wet-sieving and decanting technique (Gerdemann and Nicolson, 1963). The plant growth promoting rhizobacteria (PGPR) and the mycorhiza (My) treatments were used at the rate of $85 \mathrm{ml}$ for each palm.

TABLE 1. Mechanical (a) and chemical (b) analyses as well as the other properties of the experimental soil (soil:water extract 1:20) as well as the chemical analysis of the irrigation water (c) used during the two growing seasons, 2013-2014.

\begin{tabular}{|c|c|c|c|c|c|c|c|c|c|c|c|}
\hline \multirow[b]{2}{*}{ Seas } & \multicolumn{4}{|c|}{ a) Mechanical analysis\% } & \multicolumn{7}{|c|}{ b) Chemical analysis of the soil } \\
\hline & $\begin{array}{l}\text { Coarse+ } \\
\text { fine } \\
\text { Sand }\end{array}$ & Silt & Clay & \begin{tabular}{|} 
Soil \\
Textur
\end{tabular} & \multicolumn{4}{|c|}{ Cations meq/100g } & \multicolumn{3}{|c|}{$\begin{array}{c}\text { Anions meq/ } \\
100 \mathrm{~g}\end{array}$} \\
\hline 2013 & 69.3 & 20.2 & 10.44 & \multirow{3}{*}{$\begin{array}{l}\text { Sandy } \\
\text { Loamy } \\
\text { soil }\end{array}$} & & $\mathrm{Ca}$ & $\mathrm{Mg}$ & $\mathrm{Na}$ & $\mathrm{K}$ & $\mathrm{SO} 4$ & $\mathrm{Cl}$ \\
\hline & & & & & 6.07 & 3.64 & 12.92 & 0.35 & 0.18 & 9.25 & 13.55 \\
\hline 2014 & 68.5 & 21.0 & 10.51 & & 6.05 & 3.57 & 12.53 & 0.27 & 0.17 & 8.63 & 13.56 \\
\hline
\end{tabular}

\begin{tabular}{|c|c|c|c|c|c|c|c|c|c|c|c|c|c|}
\hline & & & b) Che & mical & aly & is of & the $s$ & oil (co & ont.) & & & & \\
\hline Seaconl & FC & H & Sp\% & $\mathbf{C a C}$ & $\begin{array}{c}\text { Orga } \\
\text { nic }\end{array}$ & Mic & cro el & emrn & ts (pp & & & $\begin{array}{l}\text { vailal } \\
\text { (ppm }\end{array}$ & \\
\hline peasont & De asm & pn & DF\% & $\mathbf{O}_{3} \%$ & \begin{tabular}{|c}
$\begin{array}{c}\text { matte } \\
\text { r\% }\end{array}$ \\
\end{tabular} & $\mathrm{Fe}$ & Zn & $\begin{array}{l}\mathbf{M} \\
\mathbf{n} \\
\end{array}$ & $\mathrm{Cu}$ & B & $\mathbf{N}$ & $\mathbf{P}$ & $\mathbf{K}$ \\
\hline 2013 & 4.42 & 8.03 & 43.21 & 2.67 & 0.59 & 17.14 & 3.23 & 10.26 & 2.24 & 9.15 & $24 . .25$ & 3.78 & 24.23 \\
\hline 2014 & 4.4 & 8.31 & 41.34 & 2.6 & 0.47 & 16.21 & 3.35 & 9.38 & 2.29 & 9.11 & 30.21 & 3.7 & 19.34 \\
\hline c) $\mathrm{C}$ & hemical : & analy & is of $t$ & he irr & gation & wate & r use & d meq & & & & & \\
\hline Soocon & EC & & An & ions & & & Cat & ions & & & & & \\
\hline | & $\mathrm{dsm}^{-1}$ & $\mathrm{CO}_{3}$ & $\mathrm{HCO}_{3-}$ & $\mathrm{SO}_{4}^{-}$ & $\mathrm{Cl}^{-}$ & $\mathrm{Na}^{+}$ & $\mathbf{K}^{+}$ & $\mathrm{Ca}^{++}$ & $\mathrm{Mg}^{++}$ & & & & \\
\hline 2013 & 5.64 & $\ldots$ & 1.28 & 26.49 & 25.02 & 50.63 & 0.18 & 0.75 & 1.24 & & & & \\
\hline 2014 & 5.52 & $\ldots$ & 1.17 & 25.99 & 26 & $\$ 1.03$ & 0.17 & 0.71 & 1.25 & & & & \\
\hline
\end{tabular}

\section{Putrescine treatments}

Concentrations of putrescine (Sigma Aldrich) denoted 2.5 and $5 \mathrm{mM}$ in addition to distilled water as a control were applied to the soil in circle holes around each palm tree with $50 \mathrm{~cm}$ depth and $70 \mathrm{~cm}$ distance from the plant truck.

\section{Experiment design}

108 female palm trees, 10 years old, similar vigor, height, pollen source and planted at $7 \times 7 \mathrm{~m}$ were selected. The trees were arranged in a randomized 
complete block design with 3 replicates (three trees for each) and irrigated using furrow irrigation system.

The experiment included control, plant growth promoting rhizobacteria (PGPR), Mycorhiza (My), PGPR+Mycorhiza (My) with or Without Putresine (Put). The application of all treatments took place at three times with 20 days interval. The $1^{\text {st }}$ time was done at blooming and the beginning of set stage at April 15. Other agricultural practices were applied as recommended by Ministry of Agric., Egypt.

\section{Sampling and data recorded}

Leaflets of the $4^{\text {th }}$ full expanded leaf from the plant tip were taken at Kimri stage (30 June) for chemical analysis. Photosynthetic pigments were extracted and determined (Wettestein, 1957). Nitrogen, phosphorus and potas sium concentrations were detected according to the methods described by Bremner \& Muluaney (1982), Olsen \& Sommers (1982) and Chapman \& Pratt (1982) respectively. Moreover, certain organic osmolytes components with have possible involvement of the antioxidant systemin relation to salt tolerance were analyzed, Total free amino acids (TAA) and total sugars (TS) were extracted from the plant material by $80 \%$ ethanol. TAA was determined spectrophotometrically by the methods of Dubey and Rani (1989a, b) and total sugars were determined by phenol-sulphuric acid method as described by Sadasivam and Manickam (1996). Glutathione Reductase (EC 1.8.1.7) (GR) activity was measured according to Foyer and Halliwell (1976). One enzyme unit is defined as $\mu \mathrm{moL} \mathrm{mL}{ }^{-1}$ oxidized Glutathione per min. Ascorbate Peroxidase (EC.1.11.1.11), (APOX) activity was assayed according to Nakano and Asada (1981). One enzyme unit is defined as $\mu \mathrm{moL} \mathrm{mL} \mathrm{m}^{-1}$ oxidized ascorbate per min. Superoxide Dismutase (EC 1.15.1.1) (SOD) activity was assayed based on the method of Beauchamp and Fridovich (1971) and the specific enzyme activity was expressed as units $\mathrm{mg}^{-1}$ protein $\mathrm{g} \mathrm{FW}$. Lipid peroxidation was measured as the quantum of thiobarbituric acid reactive susbstances (TBARS) determined by the thiobarbituric acid (TBA) reaction as described by Borsani et al., (2001). The concentration of TBARS was calculated (Groppa et al., 2001 and 2003). Diamine oxidase (DAO, EC 1.4.3.6) and polyamine oxidase (PAO, 1.4.3.4) activtities were prepared as described by (Aribaud et al., 1994 and Faive-Rampant et al., 2000) and determined using $\mathrm{H}_{2} \mathrm{O}_{2}$ - D method (Nag et al., 2000). Endogenous phytohormone quantity determined using high-performance liquid chromatography (HPLE) according to Koshioka et al., (1983) for auxins (IAA), gibberelic acid $\left(\mathrm{GA}_{3}\right)$ and Abcisic acid (ABA) while cytokinins were detected according to Nicander et al., (1993).

The harvesting took place periodically for six times, 15 days intervals from August $15^{\text {th }}$ till the November $1^{\text {st }}$. At the end of harvesting time, cumulative fruit yield was measured during the two growing seasons. At the third harvesting time (Sept. $15^{\text {th }}$ ) fruit component of total sugars and carbohydrates (Amberger, 1954), total soluble solid, (TSS) using a hand refractometer, Vitamin C (AOAC, 1995) as well as total nutrients of N, $\mathrm{P}$ and $\mathrm{K}$ (as previously mentioned) were estimated. Total crude protein \% was calculated by multiplying total nitrogen x 6.25 (AOAC, 1995). 
Statistical analysis

All data were subjected to analysis of variance (Snedecor and Cochran, 1980) using SAS system (2003).

\section{Results and Discussion}

\section{Physoligical parameters \\ Photosynthetic pigments}

Table 2 shows that $\mathrm{Chl} \mathrm{a}, \mathrm{b}$ and carotenoids concentrations were increased due to PGPR, My and put application compared to the control during the two growing seasons. The combination treatments between PGPR and My gave the highest values in the presence of put at $2.5 \mathrm{mM}$ compared with the individual application and the control. Increase of Chl(s) and carotenoids concentration may be enhanced photosynthesis efficiency and that is a good explain to the increasing of date palm productivity. In addition, this enhancement could be indication for expectable high fruit yield quality when date palm irrigated with saline water and grown in the reclaimed salinized soil. In this respect, Heidari and Golpayegani (2012) found that inoculation with rhizobacteria could be efficiently used to improve growth, antioxidant status and photosynthesis pigments under water stress. Moreover, Makela et al, (2000) used My to protectively photosynthetic machinery of plant organelles by stabilizing the ultrastructure of the chloroplast. PSח reaction centers and maintaining the oxygen - evolving machineries. Similarly, the increase in photosynthetic pigments in the plants treated with exogenous My may be due to its effects on lowering membrane damage, better photosynthesis rate, improved leaf water potential and greater shoot dry weight (Wahid and Shabbir, 2005). Stabilizes pigments as well as prevents water oxidation and photo oxidation were also detected due to My and putresine application (Cha-um et al., 2006). They added that stabilization of Chl a,b and carotenoids in light energy capture as required for photosynthesis. Putresine may assume a different role in non-photosynthetic organs vs. photosynthetic ones since it acculated to high levels in roots of the salt-resistant plants compared with the salt sensitive one while an apposite trend was recorded in the shoots (Cowley and Walters, 2002).

\section{NPK minerals and Organic osmolytes}

Table 3 show the effects of plant growth promoting rhizobacteria (PGPR), and/or Mycorhiza (My) with or without Putresine amine on concentrations of N,P,K, as well as total sugars, total free amino acids. The data in the table indicated that PGPR, My in the presence or absence of put significantly increased concentrations of N, P and $\mathrm{K}$, total sugars, and total free amino acids in the leaflets of date palm in both seasons. Moreover put at $2.5 \mathrm{mM}$ alone or incombination with other treatments gave the best significantly highest values of total sugars, total free amino acids. Similar results were reported by Bhatti et al. (2013) who found that the content of total sugars and some of the bioconstituets may be considered as a direct result for high rate of photosynthesis with great efficiency that was preceded with large photosynthetic area and high content of photosynthetic pigments. Liu and Huang (2000) suggested that 
high carbohydrate available (glucose and sucrose) during stress condition are represents of important physiological traits associated with stress tolerance. In addition accumulation of amino acids and sugars are necessary to regulate os motic activities and protectcellular structures fromstress condition by maintaining the cell water balance and membrane stability. Ahmed and Kibret (2014) suggested that PGPR promote plant growth directly by facilitating resource acquisition nitrogen, phosphorus and other es sential elements. Moreover, changes induced by salt stress in sugars and amino acids related to synthes is of polyamines (glutamate, arginine and proline) and polyamines putrescine were reported in leaves of a salt-sensitive tomato species (Lycopersecon esculeatum, Mill) and its wild salt tolerant reactive species (L. pennelli Correll) Darcy in light and dark after short-term exposure (Zapata et al., 2004). Therefore polyamines have been suggested to contrast oxidative damage in plants as indicated in our results.

TABLE 2. Effect of plant growth promoting rhizobacteria (PGPR) Mycorhiza (My), Putresine amine alone or in combination on chlorophyll $a, b$ and carotenoids concentrations (mg/g Fwt) in the leaflets of the $4^{\text {th }}$ full expanded leaffrom the plant tip of date palm (zaghloul cv.) irrigated with saline water and grown in the reclaimed saline soil during the two growing seasons (2013 and 2014).

\begin{tabular}{|c|c|c|c|c|c|c|c|c|}
\hline \multirow{4}{*}{ Freatments } & \multicolumn{8}{|c|}{ photosynthetic Pigments mg/100g F.Wt. } \\
\hline & \multicolumn{8}{|c|}{ Growing seasons } \\
\hline & \multicolumn{4}{|c|}{ First season 2013} & \multicolumn{4}{|c|}{ Second season 2014} \\
\hline & Chl a & Chl b & Ch $\mathbf{a}+\mathbf{b}$ & Car. & Chl a & Chl b & $\mathbf{C h} \mathbf{a}+\mathbf{b}$ & Car. \\
\hline Control & $\begin{array}{l}27.6 \pm \\
0.63 \mathrm{f}\end{array}$ & $\begin{array}{l}9.64 \pm \\
0.42 \mathrm{f}\end{array}$ & $\begin{array}{c}38.54 \pm \\
0.91 \mathrm{f}\end{array}$ & $\begin{array}{c}13.26 \pm \\
0.44 \mathrm{e}\end{array}$ & $\begin{array}{l}27.9 \pm \\
0.73 \mathrm{e}\end{array}$ & $\begin{array}{l}9.95 \pm \\
0.41 \mathrm{f}\end{array}$ & $\begin{array}{c}38.85 \pm \\
1.65 \mathrm{~g}\end{array}$ & $\begin{array}{c}13.53 \pm \\
0.47 \mathrm{~d}\end{array}$ \\
\hline $2.5 \mathrm{mM}$ put & $\begin{array}{l}35.4 \pm \\
0.71 \mathrm{c}\end{array}$ & $\begin{array}{c}11.62 \pm \\
0.73 \mathrm{c}\end{array}$ & $\begin{array}{c}48.02 \pm \\
1.90 \mathrm{c}\end{array}$ & $\begin{array}{l}14.8 \pm \\
0.53 \mathrm{c}\end{array}$ & $\begin{array}{l}35.8 \pm \\
0.83 \mathrm{c}\end{array}$ & $\begin{array}{l}11.62 \pm \\
0.49 \mathrm{c}\end{array}$ & $\begin{array}{c}46.02 \pm \\
1.78 \mathrm{c}\end{array}$ & $\begin{array}{l}14.8 \pm \\
10.49 \mathrm{c}\end{array}$ \\
\hline $5 \mathrm{mM}$ put & $\begin{array}{l}32.7 \pm \\
0.84 \mathrm{e}\end{array}$ & $\begin{array}{c}10.75 \pm \\
0.42 \mathrm{e}\end{array}$ & $\begin{array}{c}43.75 \pm \\
1.82 \mathrm{e}\end{array}$ & $\begin{array}{l}14.2 \pm \\
0.57 \mathrm{c}\end{array}$ & $\begin{array}{l}33.4 \pm \\
0.61 \mathrm{~d}\end{array}$ & $\begin{array}{l}10.86 \pm \\
0.47 \mathrm{e}\end{array}$ & $\begin{array}{c}43.75 \pm \\
1.68 \mathrm{f}\end{array}$ & $\begin{array}{l}14.2 \pm \\
0.51 \mathrm{c}\end{array}$ \\
\hline PGPR & $\begin{array}{l}33.2 \pm \\
0.72 \mathrm{e}\end{array}$ & $\begin{array}{c}11.62 \pm \\
0.48 \mathrm{c}\end{array}$ & $\begin{array}{c}44.46 \pm \\
1.86 \mathrm{e}\end{array}$ & $\begin{array}{l}14.7 \pm \\
0.56 \mathrm{c}\end{array}$ & $\begin{array}{l}33.6 \pm \\
0.77 \mathrm{~d}\end{array}$ & $\begin{array}{c}11.92 \pm \\
0.53 \mathrm{c}\end{array}$ & $\begin{array}{c}44.46 \pm \\
1.73 \mathrm{e}\end{array}$ & $\begin{array}{l}14.7 \pm \\
0.53 \mathrm{c}\end{array}$ \\
\hline $\begin{array}{l}\text { PGPR+ } \\
2.5 \mathrm{mMput}\end{array}$ & $\begin{array}{l}38.3 \pm \\
0.88 \mathrm{~b}\end{array}$ & $\begin{array}{c}12.81 \pm \\
0.44 \mathrm{~b}\end{array}$ & $\begin{array}{c}51.11 \pm \\
1.84 \mathrm{~b}\end{array}$ & $\begin{array}{l}16.3 \pm \\
0.57 \mathrm{~b}\end{array}$ & $\begin{array}{l}39.4 \pm \\
0.88 b\end{array}$ & $\begin{array}{c}13.21 \pm \\
0.56 \mathrm{~b}\end{array}$ & $\begin{array}{c}51.11 \pm \\
1.72 \mathrm{~b}\end{array}$ & $\begin{array}{l}16.3 \pm \\
0.52 b\end{array}$ \\
\hline $\begin{array}{l}\text { PGPR+ } \\
5 \text { mM put }\end{array}$ & $\begin{array}{l}34.9 \pm \\
0.61 \mathrm{~d}\end{array}$ & $\begin{array}{c}11.92 \pm \\
0.58 \mathrm{c}\end{array}$ & $\begin{array}{c}46.82 \pm \\
1.87 \mathrm{~d}\end{array}$ & $\begin{array}{l}15.2 \pm \\
0.63 \mathrm{~b}\end{array}$ & $\begin{array}{l}35.8 \pm \\
0.87 \mathrm{c}\end{array}$ & $\begin{array}{l}12.68 \pm \\
0.57 \mathrm{~b}\end{array}$ & $\begin{array}{c}46.82 \pm \\
1.68 \mathrm{~d}\end{array}$ & $\begin{array}{l}15.2 \pm \\
0.55 b\end{array}$ \\
\hline My & $\begin{array}{l}34.1 \pm \\
0.53 \mathrm{~d}\end{array}$ & $\begin{array}{c}11.21 \pm \\
0.43 \mathrm{~d}\end{array}$ & $\begin{array}{c}45.31 \pm \\
1.82 \mathrm{e}\end{array}$ & $\begin{array}{l}15.3 \pm \\
0.64 \mathrm{~b}\end{array}$ & $\begin{array}{l}34.7 \pm \\
0.79 \mathrm{c}\end{array}$ & $\begin{array}{c}11.82 \pm \\
0.51 \mathrm{c}\end{array}$ & $\begin{array}{c}45.31 \pm \\
1.71 \mathrm{e}\end{array}$ & $\begin{array}{l}15.3 \pm \\
0.56 \mathrm{~b}\end{array}$ \\
\hline \begin{tabular}{|l|} 
My $+2.5 \mathrm{mM}$ \\
put
\end{tabular} & $\begin{array}{l}35.1 \pm \\
0.75 \mathrm{c}\end{array}$ & $\begin{array}{c}12.12 \pm \\
0.49 \mathrm{~b}\end{array}$ & $\begin{array}{c}47.32 \pm \\
1.91 \mathrm{~d}\end{array}$ & $\begin{array}{l}15.8 \pm \\
0.61 \mathrm{~b}\end{array}$ & $\begin{array}{l}35.8 \pm \\
0.78 \mathrm{c}\end{array}$ & $\begin{array}{c}12.14 \pm \\
0.55 \mathrm{~b}\end{array}$ & $\begin{array}{c}47.22 \pm \\
1.70 \mathrm{c}\end{array}$ & $\begin{array}{l}15.8 \pm \\
0.52 b\end{array}$ \\
\hline My + & $34.3 \pm$ & $11.65 \pm$ & $\begin{array}{r}45.95 \pm \\
1.93 d\end{array}$ & $15.1 \pm$ & $35.2 \pm$ & $12.15 \pm$ & $45.95 \pm$ & $15.1 \pm$ \\
\hline \begin{tabular}{|l}
$5 \mathrm{mM}$ put \\
$\mathrm{PGPR}+$
\end{tabular} & $\frac{0.61 \mathrm{~d}}{34.8 \pm}$ & \begin{tabular}{|c|}
$0.53 \mathrm{c}$ \\
$11.35 \pm$
\end{tabular} & \begin{tabular}{|c|}
$1.93 \mathrm{~d}$ \\
$46.16 \pm$
\end{tabular} & $\frac{0.59 \mathrm{~b}}{15.3 \pm}$ & $\frac{0.87 \mathrm{c}}{34.8 \pm}$ & $\frac{0.57 \mathrm{~b}}{11.74 \pm}$ & $\frac{1.73 \mathrm{~d}}{46.16 \pm}$ & $\begin{array}{c}0.57 \mathrm{~b} \\
15.3 \pm\end{array}$ \\
\hline My & $0.66 \mathrm{c}$ & $0.54 \mathrm{c}$ & $1.83 \mathrm{~d}$ & $0.62 b$ & $0.85 \mathrm{c}$ & $0.53 \mathrm{c}$ & $1.75 \mathrm{~d}$ & $0.54 b$ \\
\hline $\begin{array}{l}\text { PGPR+My+ } \\
2.5 \mathrm{mMput}\end{array}$ & $\begin{array}{l}47.6 \pm \\
0.82 \mathrm{a}\end{array}$ & $\begin{array}{c}15.41 \pm \\
0.62 \mathrm{a}\end{array}$ & $\begin{array}{c}63.01 \pm \\
1.92 \mathrm{a}\end{array}$ & $\begin{array}{l}19.7 \pm \\
0.64 \mathrm{a}\end{array}$ & $\begin{array}{r}48.9 \pm \\
0.84 \mathrm{a}\end{array}$ & $\begin{array}{c}15.82 \pm \\
0.59 \mathrm{a}\end{array}$ & $\begin{array}{c}63.01 \pm \\
1.77 \mathrm{a}\end{array}$ & $\begin{array}{l}19.7 \pm \\
0.61 \mathrm{a}\end{array}$ \\
\hline PGPR+My+ & $35.3 \pm$ & $12.24 \pm$ & $47.54 \pm$ & $15.8 \pm$ & $35.7 \pm$ & $12.76 \pm$ & $47.54 \pm$ & $15.8 \pm$ \\
\hline $5 \mathrm{mM}$ put & $0.77 \mathrm{c}$ & 0.64 & $1.94 \mathrm{~d}$ & $0.65 b$ & $0.87 \mathrm{c}$ & $0.54 \mathrm{~b}$ & $1.69 \mathrm{c}$ & $0.52 b$ \\
\hline
\end{tabular}

Mean values \pm SD followed by different letter are significantly different by ANOVA at 0.05 level. 
TABLE 3. Effects of plant growth promoting rhizobacteria (PGPR), Mycorhiza (My), Putresine amine alone or in combination on concentrations of $\mathbf{N}, \mathbf{P}, \mathrm{K}$, as well as total sugars and total free amino acids in the leaflets of the $4^{\text {th }}$ leaf from the plant tip of date palm (Phoenix dactylifera $\mathrm{L}$.) irrigated with saline water and grown in reclaimed saline soil during the two growing seasons (2013 and 2014).

\begin{tabular}{|c|c|c|c|c|c|c|c|c|c|c|}
\hline \multirow{4}{*}{ Treatments } & \multicolumn{10}{|c|}{ Growing seasons } \\
\hline & \multicolumn{5}{|c|}{ First season 2013} & \multicolumn{5}{|c|}{ Second season 2014} \\
\hline & \multicolumn{3}{|c|}{$\begin{array}{l}\text { Nutrients } \\
\text { mg/g D.wt. }\end{array}$} & \multirow{2}{*}{$\begin{array}{l}\text { Total } \\
\text { sugars } \\
\text { mg/g } \\
\text { F.wt. }\end{array}$} & \multirow{2}{*}{\begin{tabular}{|l} 
Total \\
free \\
amino \\
acids \\
mg/g \\
F.wt.
\end{tabular}} & \multicolumn{3}{|c|}{$\begin{array}{l}\text { Nutrients } \\
\text { mg/g D.wt. }\end{array}$} & \multirow{2}{*}{$\begin{array}{c}\text { Total } \\
\text { sugars } \\
\text { mg/g } \\
\text { F.wt. }\end{array}$} & \multirow{2}{*}{$\begin{array}{l}\text { Total } \\
\text { free } \\
\text { amino } \\
\text { acids } \\
\text { mg/g } \\
\text { F.wt. }\end{array}$} \\
\hline & $\mathbf{N}$ & $\mathbf{P}$ & $\mathbf{K}$ & & & $\mathbf{N}$ & $\mathbf{P}$ & $\mathbf{K}$ & & \\
\hline \multirow[t]{2}{*}{ Control } & $27.2 \pm$ & $2.3 \pm$ & $31.1 \pm$ & $16.2 \pm$ & $8.7 \pm$ & $27.7 \pm$ & $2.4 \pm$ & $33.2 \pm$ & $16.4 \pm$ & $9.3 \pm$ \\
\hline & $1.05 \mathrm{~d}$ & $0.51 \mathrm{e}$ & $1.23 \mathrm{f}$ & $0.43 \mathrm{f}$ & $0.68 \mathrm{e}$ & $0.63 \mathrm{f}$ & $1.37 \mathrm{~d}$ & $1.21 \mathrm{~d}$ & $1.53 \mathrm{e}$ & $1.03 \mathrm{~d}$ \\
\hline \multirow[t]{2}{*}{$2.5 \mathrm{mM}$ put } & $31.4 \pm$ & $3.9 \pm$ & $40.6 \pm$ & $21.4 \pm$ & $12.7 \pm$ & $31.8 \pm$ & $4.0 \pm$ & $41.4 \pm$ & $21.0 \pm$ & $12.7 \pm$ \\
\hline & $1.12 \mathrm{~b}$ & $0.42 \mathrm{c}$ & $1.31 \mathrm{c}$ & $0.43 \mathrm{c}$ & $0.87 \mathrm{c}$ & $1.02 \mathrm{c}$ & $0.48 b$ & $1.01 \mathrm{~b}$ & $1.13 b$ & $0.67 \mathrm{c}$ \\
\hline \multirow[t]{2}{*}{$5 \mathrm{mM}$ put } & $28.9 \pm$ & $3.1 \pm$ & $39.1 \pm$ & $19.4 \pm$ & $11.2 \pm$ & $29.8 \pm$ & $3.4 \pm$ & $39.2 \pm$ & \begin{tabular}{|l|}
$19.9 \pm$ \\
\end{tabular} & $11.6 \pm$ \\
\hline & $1.21 \mathrm{c}$ & $0.53 \mathrm{~d}$ & $1.32 \mathrm{c}$ & $0.51 \mathrm{~d}$ & $0.76 \mathrm{c}$ & $1.20 \mathrm{~d}$ & $0.57 \mathrm{c}$ & $1.02 \mathrm{c}$ & $1.21 \mathrm{c}$ & $0.68 \mathrm{c}$ \\
\hline \multirow[t]{2}{*}{ PGPR } & $28.7 \pm$ & $3.2 \pm$ & $38.4 \pm$ & $18.7 \pm$ & $10.7 \pm$ & $28.9 \pm$ & $3.6 \pm$ & $38.6 \pm$ & $18.6 \pm$ & $10.9 \pm$ \\
\hline & $1.32 \mathrm{c}$ & $0.57 \mathrm{~d}$ & $1.05 \mathrm{~d}$ & $0.78 \mathrm{e}$ & $0.83 \mathrm{~d}$ & $1.02 \mathrm{e}$ & $0.59 \mathrm{c}$ & $1.15 \mathrm{c}$ & $0.85 \mathrm{~d}$ & $0.86 \mathrm{~d}$ \\
\hline PGPR+ & $34.8 \pm$ & $4.3 \pm$ & $42.1 \pm$ & $22.7 \pm$ & $13.4 \pm$ & $34.2 \pm$ & $4.5 \pm$ & $42.3 \pm$ & $22.1 \pm$ & $13.6 \pm$ \\
\hline $2.5 \mathrm{mMput}$ & $1.15 \mathrm{a}$ & $0.62 b$ & $1.07 \mathrm{~b}$ & $0.45 b$ & $0.76 b$ & $1.17 \mathrm{~b}$ & $0.72 b$ & $1.16 \mathrm{~b}$ & $1.05 \mathrm{~b}$ & $0.78 b$ \\
\hline PGPR+ & $32.4 \pm$ & $3.8 \pm$ & $38.5 \pm$ & $20.3 \pm$ & $11.8 \pm$ & $32.8 \pm$ & $3.9 \pm$ & $38.6 \pm$ & $20.4 \pm$ & $11.8 \pm$ \\
\hline $5 \mathrm{mM}$ put & $1.18 \mathrm{~b}$ & $0.54 \mathrm{c}$ & $1.13 \mathrm{~d}$ & $0.65 \mathrm{c}$ & $0.92 \mathrm{c}$ & $1.08 \mathrm{c}$ & $0.56 \mathrm{~b}$ & $1.03 \mathrm{c}$ & $1.07 \mathrm{c}$ & $0.81 \mathrm{c}$ \\
\hline \multirow[t]{2}{*}{ My } & $31.5 \pm$ & $3.8 \pm$ & $37.9 \pm$ & $19.8 \pm$ & $11.2 \pm$ & $31.2 \pm$ & $3.9 \pm$ & $37.9 \pm$ & $19.8 \pm$ & $11.5 \pm$ \\
\hline & $1.17 \mathrm{~b}$ & $0.74 \mathrm{c}$ & $1.07 \mathrm{e}$ & $0.86 \mathrm{~d}$ & $0.83 c$ & $1.09 \mathrm{c}$ & $0.83 b$ & $1.17 \mathrm{c}$ & $0.76 \mathrm{c}$ & $0.86 \mathrm{c}$ \\
\hline \multirow{2}{*}{$\begin{array}{l}\text { My }+2.5 \mathrm{mM} \\
\text { put }\end{array}$} & $32.9 \pm$ & $3.8 \pm$ & $40.3 \pm$ & $21.2 \pm$ & $12.3 \pm$ & $31.5 \pm$ & $3.7 \pm$ & $40.3 \pm$ & $21.3 \pm$ & $12.6 \pm$ \\
\hline & $1.42 \mathrm{~b}$ & $.75 \mathrm{c}$ & $1.36 \mathrm{c}$ & $0.54 \mathrm{c}$ & $0.94 \mathrm{c}$ & $1.12 \mathrm{c}$ & $0.65 \mathrm{c}$ & $1.36 \mathrm{~b}$ & $1.14 \mathrm{~b}$ & $0.85 c$ \\
\hline \multirow{2}{*}{$\begin{array}{l}\text { My + } 5 \mathrm{mM} \\
\text { put }\end{array}$} & $30.7 \pm$ & $3.5 \pm$ & $39.2 \pm$ & $19.6 \pm$ & $12.1 \pm$ & $30.7 \pm$ & $3.6 \pm$ & $39.3 \pm$ & $19.7 \pm$ & $12.4 \pm$ \\
\hline & $1.32 \mathrm{~b}$ & $0.68 \mathrm{c}$ & $1.16 \mathrm{c}$ & $0.68 \mathrm{~d}$ & $0.85 \mathrm{c}$ & $1.02 \mathrm{~d}$ & $0.78 \mathrm{c}$ & $1.06 \mathrm{c}$ & $0.69 \mathrm{c}$ & $0.65 \mathrm{c}$ \\
\hline \multirow[t]{2}{*}{ PGPR + My } & $32.5 \pm$ & $3.2 \pm$ & $38.6 \pm$ & $20.2 \pm$ & $11.2 \pm$ & $30.8 \pm$ & $3.4 \pm$ & $38.7 \pm$ & $20.5 \pm$ & $11.3 \pm$ \\
\hline & $1.27 \mathrm{~b}$ & $0.74 \mathrm{c}$ & $1.06 \mathrm{~d}$ & $0.88 \mathrm{c}$ & $0.93 \mathrm{c}$ & $1.21 \mathrm{~d}$ & $0.70 \mathrm{c}$ & $1.08 \mathrm{c}$ & $0.87 \mathrm{c}$ & $0.92 \mathrm{c}$ \\
\hline \begin{tabular}{|l|}
$\mathrm{PGPR}+\mathrm{My}+$ \\
\end{tabular} & $35.3 \pm$ & $5.0 \pm$ & $43.8 \pm$ & $23.5 \pm$ & $14.1 \pm$ & $35.9 \pm$ & $5.3 \pm$ & $43.9 \pm$ & $23.8 \pm$ & $14.6 \pm$ \\
\hline 2.5mMput & $1.12 \mathrm{a}$ & $0.34 \mathrm{a}$ & $1.08 \mathrm{a}$ & $0.92 \mathrm{a}$ & $0.86 \mathrm{a}$ & $1.01 \mathrm{a}$ & $0.37 \mathrm{a}$ & $1.18 \mathrm{a}$ & $0.52 \mathrm{a}$ & $0.75 \mathrm{a}$ \\
\hline PGPR+My+ & $31.9 \pm$ & $3.4 \pm$ & $40.3 \pm$ & $21.8 \pm$ & $12.2 \pm$ & $31.7 \pm$ & $3.6 \pm$ & $40.2 \pm$ & $21.7 \pm$ & $12.3 \pm$ \\
\hline $5 \mathrm{mMput}$ & $1.14 \mathrm{~b}$ & $0.48 \mathrm{c}$ & $1.12 \mathrm{c}$ & $0.87 \mathrm{c}$ & $0.74 \mathrm{c}$ & $1.34 \mathrm{~b}$ & $0.58 \mathrm{c}$ & $1.14 \mathrm{~b}$ & $0.87 \mathrm{~b}$ & $078 \mathrm{c}$ \\
\hline
\end{tabular}

Ascorbate peroxidase (APOX) glutathione reductase (GR) and superoxide dismutase (SOD) activities

The activities of APOX, GR, and SOD were decreased due to the application of PGPR, My and/or Put (Table 4). Put showed an additive effects to that of PGPR and My in combined treatments. The lowest activity levels were recorded in Bio+My in the presence of put at $2.5 \mathrm{mM}$ (Table 4). No significant differences were observed in the activates of APOX, GR, and SOD when $5 \mathrm{mM}$ put was used compared to the control. Similar results were reported by Tang and Newt on (2005), who reported that amines reduce salt-induced oxidative damage by increasing the activates of antioxidant enzymes and decreasing lipid peroxidation in Uirginia pine. They added that APOX, GR, and SOD were associated with the oxidative damage of the enzymatic defense system caused by salt stress.

Egypt. J. Hort. Vol. 42, No. 1 (2015) 
TABLE 4. Effects of plant growth promoting rhizobacteria (PGPR), Mycorhiza (My), Putresine amine alone or in combination on the activities of antioxidant enzyme, ascorbate peroxidase (APOX), glutathione reductase (GR) and superoxide dismutase (SOD) in the leaflets of the $4^{\text {th }}$ leaf from the plant tip of date palm (Phoenix dactylifera $\mathrm{L}$.) irrigated with saline water and grown in reclaimed saline soil during the two growing seasons (2013 and 2014).

\begin{tabular}{|c|c|c|c|c|c|c|}
\hline \multirow{4}{*}{ Treatments } & \multicolumn{6}{|c|}{ Growing seasons } \\
\hline & \multicolumn{3}{|c|}{ First season 2013} & \multicolumn{3}{|c|}{ Second season 2014} \\
\hline & APOX & GR & SOD & APOX & GR & SOD \\
\hline & $\mu / \mathrm{g}$ Fwt. & $\mu / 100 g$ Fwt. & $\mu / 100 g$ Fwt. & $\mu / g$ Fwt. & $\mu / 100 \mathrm{~g}$ Fwt. & $\mu / 100 g$ Fwt. \\
\hline Control & $18.76 \pm 0.571 \mathrm{~d}$ & $13.96 \pm 0.41 \mathrm{~d}$ & $13.61 \pm 0.57 \mathrm{e}$ & $18.74 \pm 0.71 \mathrm{f}$ & $14.21 \pm 0.81 \mathrm{f}$ & $12.41 \pm 0.73 \mathrm{~d}$ \\
\hline $2.5 \mathrm{mM}$ put & $11.63 \pm 0.84 \mathrm{~b}$ & $10.86 \pm 0.63 b$ & $9.96 \pm 0.73 b$ & $11.42 \pm 0.67 \mathrm{~b}$ & $12.26 \pm 0.80 \mathrm{c}$ & $9.62 \pm 0.74 \mathrm{~b}$ \\
\hline $5 \mathrm{mM}$ put & $18.77 \pm 0.62 d$ & $13.12 \pm 0.583$ & $11.32 \pm 0.72 \mathrm{c}$ & $18.17 \pm 0.81 \mathrm{e}$ & $13.12 \pm 0.78 \mathrm{e}$ & $11.62 \pm 0.72 \mathrm{c}$ \\
\hline PGPR & $16.30 \pm 0.54 \mathrm{~d}$ & $13.37 \pm 0.51 \mathrm{c}$ & $12.38 \pm 0.81 \mathrm{~d}$ & $16.01 \pm 0.72 \mathrm{~d}$ & $13.48 \pm 0.75 \mathrm{e}$ & $12.32 \pm 0.91 \mathrm{~d}$ \\
\hline PGPR+2.5 mMput & $10.76 \pm 0.61 \mathrm{a}$ & $10.1 \pm 0.41 \mathrm{~b}$ & $9.21 \pm 0.68 \mathrm{a}$ & $10.25 \pm 0.78 \mathrm{a}$ & $11.26 \pm 0.82 b$ & $9.31 \pm 0.90 \mathrm{~b}$ \\
\hline PGPR+ $5 \mathrm{mM}$ put & $16.34 \pm 0.77 \mathrm{~d}$ & $13.39 \pm 0.35 \mathrm{c}$ & $12.46 \pm 0.81 \mathrm{~d}$ & $16.95 \pm 0.28 \mathrm{c}$ & $13.53 \pm 0.81 \mathrm{e}$ & $11.52 \pm 0.85 \mathrm{c}$ \\
\hline My & $16.53 \pm 0.64 \mathrm{c}$ & $13.57 \pm 0.59 \mathrm{c}$ & $12.48 \pm 0.75 \mathrm{~d}$ & $15.83 \pm 0.81 \mathrm{c}$ & $13.57 \pm 0.89 \mathrm{e}$ & $12.26 \pm 0.82 \mathrm{~d}$ \\
\hline $\mathrm{My}+2.5 \mathrm{mM}$ put & $15.43 \pm 0.58 \mathrm{c}$ & $13.48 \pm 0.46 \mathrm{c}$ & $12.06 \pm 0.87 \mathrm{c}$ & $15.33 \pm 0.64 \mathrm{c}$ & $13.18 \pm 0.74 \mathrm{e}$ & $11.86 \pm 0.86 \mathrm{c}$ \\
\hline $\mathrm{My}+5 \mathrm{mM}$ put & $16.87 \pm 0.54 \mathrm{c}$ & $13.26 \pm 0.64 \mathrm{c}$ & $12.64 \pm 0.81 \mathrm{~d}$ & $15.20 \pm 0.53 \mathrm{c}$ & $13.16 \pm 0.74 \mathrm{c}$ & $12.14 \pm 0.91 \mathrm{c}$ \\
\hline PGPR + My & $17.54 \pm 1.65 \mathrm{~d}$ & $13.58 \pm 0.43 \mathrm{c}$ & $13.26 \pm 0.74 \mathrm{~d}$ & $17.04 \pm 0.74 \mathrm{e}$ & $12.88 \pm 0.83 \mathrm{c}$ & $9.46 \pm 0.93 b$ \\
\hline $\mathrm{PGPR}+\mathrm{My}+2.5 \mathrm{mM}$ & $10.10 \pm 0.68 \mathrm{a}$ & $10.02 \pm 0.58 \mathrm{a}$ & $9.03 \pm 0.86 \mathrm{a}$ & $10.02 \pm 0.78 \mathrm{a}$ & $9.42 \pm 0.84 \mathrm{a}$ & $8.01 \pm 0.94 \mathrm{a}$ \\
\hline $\mathrm{PGPR}+\mathrm{My}+5 \mathrm{mM}$ & $16.26 \pm 0.78 \mathrm{c}$ & $14.26 \pm 0.58 \mathrm{~d}$ & $13.04 \pm 0.82 \mathrm{~d}$ & $16.14 \pm 0.88 \mathrm{~d}$ & $12.56 \pm 0.93 \mathrm{c}$ & $10.74 \pm 0.82 \mathrm{~b}$ \\
\hline
\end{tabular}

Mean values \pm SD followed by different letter are significantly different by ANOVA at 0.05 level.

Lipid peroxidation as well as Activates of diamine oxidase (DAO) and polyamine oxidase (PAO)

Table 5 shows that lipid peroxidation was significantly decreased due to the application of PGPR, My and/or putresine. Highest decreasing levels were detected in Bio + My +2.5 put treatment. However this parameter was not significantly decreased when $5 \mathrm{mM}$ put was used compared to the control. Therefore putresine amine protection on cell membranes was evidenced by the reduction on lipid peroxidation

The same table shows that different exogenously added PGPR, My and/or Put increased the activates of DAO and PAO in the leaflets of date palm irrigated with salinizied water and grown in the reclaimed-salinized soil. The highest activates levels were found with put $(5 \mathrm{mM})$ interacted with PGPR+My. Higher PAO activity from date palm leaflets treated with putresine amine may be resulted in liberating more hydrogen peroxidase, which in turn exert powerful physiological effects on productivity. Similar results were reported by Tang and Netwton (2005) with Viriginia pine plants. 
TABLE 5. Effects of plant growth promoting rhizobacteria (PGPR), Mycorhiza (My), Putresine amine alone or in combination on Lipid peroxidation. (Mmol/g.Fwt) as well as the activities of diamine oxidase (DAO) and amine oxidase (PAO) and activates $\left(\mathrm{mMH}_{2} \mathrm{O}_{2} / 30 \mathrm{~min} / \mathrm{gFwt}\right.$.) in the leaflets of the $4^{\text {th }}$ full expanded leaf from the plant tip of date palm (zaghloul cv.) irrigated with saline water and grown in the reclaimed saline soil during the two growing seasons ( 2013 and 2014).

\begin{tabular}{|c|c|c|c|c|c|c|}
\hline \multirow{3}{*}{ Ttreatments } & \multicolumn{6}{|c|}{ Growing seasons } \\
\hline & \multicolumn{3}{|c|}{ First season 2013} & \multicolumn{3}{|c|}{ Second season 2014} \\
\hline & $\begin{array}{c}\text { Lipid } \\
\text { peroxidation } \\
\text { Mmol/gF.Wt }\end{array}$ & $\begin{array}{c}\text { DAO } \\
\mu / g \text { Fwt. }\end{array}$ & $\begin{array}{c}\text { PAO } \\
\mu / g \text { Fwt. }\end{array}$ & $\begin{array}{c}\text { Lipid } \\
\text { peroxidation } \\
\text { Mmol/gFWt }\end{array}$ & $\begin{array}{c}\text { DAO } \\
\mu / g \text { Fwt. }\end{array}$ & $\begin{array}{c}\text { PAO } \\
\mu / g \text { Fwt. }\end{array}$ \\
\hline Control & $160.4 \pm 1.06 f$ & $1.25 \pm 0.49 \mathrm{~d}$ & $1.40 \pm 0.85 d$ & $162.1 \pm 1.08 \mathrm{f}$ & $1.41 \pm 0.35 \mathrm{e}$ & $1.56 \pm 0.32 \mathrm{f}$ \\
\hline $2.5 \mathrm{mM}$ put & $153.3 \pm 1.25 \mathrm{c}$ & $2.64 \pm 0.43 \mathrm{~b}$ & $2.82 \pm 0.68 \mathrm{~b}$ & $153.2 \pm 1.12 \mathrm{c}$ & $2.79 \pm 0.28 \mathrm{~d}$ & $3.24 \pm 0.23 \mathrm{c}$ \\
\hline $5 \mathrm{mM}$ put & $156.3 \pm 1.14 \mathrm{e}$ & $2.21 \pm 0.57 \mathrm{c}$ & $2.43 \pm 0.48 \mathrm{c}$ & $154.3 \pm 1.27 \mathrm{~d}$ & $2.23 \pm 0.620$ & $2.46 \pm 0.57 \mathrm{~d}$ \\
\hline PGPR & $157.6 \pm 1.03 \mathrm{e}$ & $2.46 \pm 0.43 \mathrm{~b}$ & $2.69 \pm 0.09 \mathrm{~b}$ & $156.6 \pm 1.24 \mathrm{e}$ & $2.46 \pm 0.43 d$ & $2.76 \pm 0.09 \mathrm{~d}$ \\
\hline PGPR+ $2.5 \mathrm{mM}$ put & $150.3 \pm 1.32 b$ & $3.48 \pm 0.48 \mathrm{a}$ & $4.13 \pm 0.45 \mathrm{a}$ & $150.1 \pm 1.41 \mathrm{~b}$ & $3.48 \pm 0.69 \mathrm{~b}$ & $4.23 \pm 0.48 \mathrm{~b}$ \\
\hline PGPR+5 $\mathrm{mM}$ put & $156.7 \pm 1.86 \mathrm{e}$ & $3.04 \pm 0.50 \mathrm{a}$ & $3.43 \pm 0.75 \mathrm{c}$ & $155.4 \pm 1.75 \mathrm{~d}$ & $3.05 \pm 0.42$ & $3.48 \pm 0.66 \mathrm{c}$ \\
\hline My & $155.6 \pm 1.95 \mathrm{~d}$ & $2.74 \pm 0.55 \mathrm{~b}$ & $2.86 \pm 0.64 \mathrm{~b}$ & $155.1 \pm 1.62 d$ & $2.76 \pm 0.72 d$ & $2.92 \pm 0.53 \mathrm{c}$ \\
\hline $\mathrm{My}+2.5 \mathrm{mM}$ put & $152.7 \pm 1.87 \mathrm{c}$ & $2.64 \pm 0.48 \mathrm{~b}$ & $2.42 \pm 0.61 \mathrm{c}$ & $152.2 \pm 1.73 \mathrm{c}$ & $2.67 \pm 0.740$ & $2.71 \pm 0.53 \mathrm{~d}$ \\
\hline $\mathrm{My}+5$ mM put & $153.8 \pm 1.65 \mathrm{c}$ & $2.14 \pm 0.54 \mathrm{c}$ & $2.02 \pm 0.26 \mathrm{c}$ & $153.2 \pm 1.57 \mathrm{c}$ & $2.16 \pm 0.54 d$ & $2.12 \pm 0.46 \mathrm{e}$ \\
\hline PGPR + My & $154.3 \pm 1.85 \mathrm{c}$ & $2.01 \pm 0.53 \mathrm{c}$ & $2.43 \pm 0.74 \mathrm{c}$ & $154.0 \pm 1.74 \mathrm{~d}$ & $2.01 \pm 0.63 \mathrm{~d}$ & $2.43 \pm 0.74 \mathrm{e}$ \\
\hline $\mathrm{PGPR}+\mathrm{My}+2.5 \mathrm{mMput}$ & $148.7 \pm 1.96 \mathrm{a}$ & $3.89 \pm 0.52 \mathrm{a}$ & $4.28 \pm 0.46 \mathrm{a}$ & $148.2 \pm 1.36 \mathrm{a}$ & $4.01 \pm 0.81 \mathrm{a}$ & $4.38 \pm 0.06 \mathrm{a}$ \\
\hline PGPR+My+ 5mM put & $153.9 \pm 1.93 \mathrm{c}$ & $2.86 \pm 0.43 \mathrm{a}$ & $2.94 \pm 0.85 b$ & $153.1 \pm 1.41 \mathrm{c}$ & $2.88 \pm 0.420$ & $2.97 \pm 0.64 c$ \\
\hline
\end{tabular}

\section{Endogenous phytohormones}

Regarding the effects of Bio, My and/or put on endogenous phytohormones, the data tabulated in Table 6 showed that all pronounces (GA, Auxin, cytokinin) were increased whereas Abscisic acid was decreased. The most effective treatment was found with Bio $+\mathrm{My}+$ put at $2.5 \mathrm{mM}$. These data could be of great influence upon different vegetative and reproduction growth. Moreover, increasing cytokinin level on the account of auxin could be in favor of increasing productivity of date palm and improving its fruit quality. Larkindale et al, (2005) found that several phytohormones including ABA and ethylene were increased under stress condition while others decreased such as GA, auxins and cytokinin. 
TABLE 6. Effects of plant growth promoting rhizobacteria (PGPR), Mycorhiza (My), Putresine amine alone or in combination on endogenous phytohormones, (GA, Auxin, cytokinin) and Abscisic acid $\left(\mathrm{mMH}_{2} \mathrm{O}_{2} / 30 \mathrm{~min} / \mathrm{gFwt}\right.$.) in leaflets of the $4^{\text {th }}$ full expanded leaf from the plant tip of date palm (zaghloul cv.) irrigated with saline water and grown in the reclaimed saline soil during the two growing seasons ( 2013 and 2014).

\begin{tabular}{|c|c|c|c|c|c|c|c|c|}
\hline \multirow[b]{3}{*}{ Treatments } & \multicolumn{8}{|c|}{ Growing seasons } \\
\hline & \multicolumn{4}{|c|}{ First season 2013} & \multicolumn{4}{|c|}{ Second season 2014} \\
\hline & $\begin{array}{l}\text { 竞 } \\
\frac{0}{0} \\
\frac{0}{0}\end{array}$ & 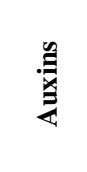 & 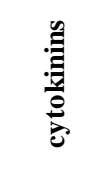 & 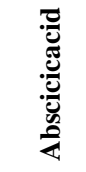 & $\begin{array}{l}\text { 竞 } \\
\frac{0}{0} \\
\frac{0}{0}\end{array}$ & $\underset{⿱ ⺌}{\mathscr{Z}}$ & 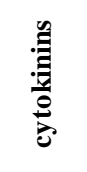 & 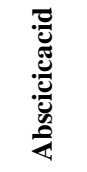 \\
\hline Control & $\begin{array}{l}30.5 \pm \\
1.03 \mathrm{~g}\end{array}$ & $\begin{array}{l}18.7 \pm \\
1.13 \mathrm{e}\end{array}$ & $\begin{array}{l}5.3 \pm \\
0.64 \mathrm{f}\end{array}$ & $\begin{array}{l}0.85 \pm \\
0.04 \mathrm{~h}\end{array}$ & $\begin{array}{l}30.7 \pm \\
1.23 \mathrm{e}\end{array}$ & $\begin{array}{l}18.9 \pm \\
1.11 \mathrm{e}\end{array}$ & $\begin{array}{r}5.8 \pm \\
0.56 \mathrm{e}\end{array}$ & $\begin{array}{l}0.83 \pm \\
0.04 \mathrm{gf}\end{array}$ \\
\hline $2.5 \mathrm{mM}$ put & $\begin{array}{l}34.1 \pm \\
1.01 \mathrm{c}\end{array}$ & $\begin{array}{l}25.6 \pm \\
1.12 \mathrm{c}\end{array}$ & $\begin{array}{l}10.7 \pm \\
0.78 \mathrm{c}\end{array}$ & $\begin{array}{l}0.64 \pm \\
0.08 \mathrm{c}\end{array}$ & $\begin{array}{l}34.7 \pm \\
1.21 \mathrm{c}\end{array}$ & $\begin{array}{l}27.1 \pm \\
1.23 \mathrm{~b}\end{array}$ & $\begin{array}{l}11.8 \pm \\
0.88 \mathrm{~b}\end{array}$ & $\begin{array}{l}0.63 \pm \\
0.07 \mathrm{c}\end{array}$ \\
\hline $5 \mathrm{mM}$ put & $\begin{array}{l}31.3 \pm \\
1.04 \mathrm{f}\end{array}$ & $\begin{array}{l}22.7 \pm \\
0.83 \mathrm{~d}\end{array}$ & $\begin{array}{c}8.5 \pm \\
0.64 \mathrm{e}\end{array}$ & $\begin{array}{l}0.72 \pm \\
0.12 \mathrm{f}\end{array}$ & $\begin{array}{l}31.9 \pm \\
1.13 \mathrm{~d}\end{array}$ & $\begin{array}{l}23.6 \pm \\
1.43 \mathrm{~d}\end{array}$ & $\begin{array}{r}8.9 \pm \\
0.67 \mathrm{c}\end{array}$ & $\begin{array}{l}0.68 \pm \\
0.08 \mathrm{e}\end{array}$ \\
\hline PGPR & $\begin{array}{l}32.5 \pm \\
1.03 \mathrm{e}\end{array}$ & $\begin{array}{l}23.9 \pm \\
1.13 \mathrm{~d}\end{array}$ & $\begin{array}{c}7.9 \pm \\
0.68 \mathrm{e}\end{array}$ & $\begin{array}{l}0.76 \pm \\
0.16 \mathrm{~g}\end{array}$ & $\begin{array}{l}33.1 \pm \\
1.02 \mathrm{c}\end{array}$ & $\begin{array}{l}24.2 \pm \\
1.23 \mathrm{~d}\end{array}$ & $\begin{array}{r}8.9 \pm \\
0.63 \mathrm{c}\end{array}$ & $\begin{array}{l}0.72 \pm \\
0.14 \mathrm{f}\end{array}$ \\
\hline \begin{tabular}{|l|} 
PGPR+2.5mM \\
put
\end{tabular} & $\begin{array}{l}35.2 \pm \\
1.21 \mathrm{~b}\end{array}$ & $\begin{array}{l}27.3 \pm \\
1.11 \mathrm{~b}\end{array}$ & $\begin{array}{l}12.9 \pm \\
0.64 b\end{array}$ & $\begin{array}{l}0.61 \pm \\
0.13 b\end{array}$ & $\begin{array}{l}36.1 \pm \\
1.24 \mathrm{~b}\end{array}$ & $\begin{array}{l}29.1 \pm \\
1.34 \mathrm{~b}\end{array}$ & $\begin{array}{l}13.9 \pm \\
0.53 \mathrm{a}\end{array}$ & $\begin{array}{l}0.61 \pm \\
0.06 \mathrm{~b}\end{array}$ \\
\hline \begin{tabular}{|l|} 
PGPR+ $5 \mathrm{mM}$ \\
put
\end{tabular} & $\begin{array}{l}33.0 \pm \\
1.06 \mathrm{c}\end{array}$ & $\begin{array}{l}24.5 \pm \\
1.03 \mathrm{c}\end{array}$ & $\begin{array}{r}8.6 \pm \\
0.68 \mathrm{e}\end{array}$ & $\begin{array}{l}0.69 \pm \\
0.17 \mathrm{c}\end{array}$ & $\begin{array}{l}33.2 \pm \\
1.14 \mathrm{c}\end{array}$ & $\begin{array}{l}25.3 \pm \\
1.13 \mathrm{c}\end{array}$ & $\begin{array}{l}11.6 \pm \\
0.68 \mathrm{~b}\end{array}$ & $\begin{array}{l}0.67 \pm \\
0.13 \mathrm{e}\end{array}$ \\
\hline My & $\begin{array}{l}33.5 \pm \\
1.13 \mathrm{c}\end{array}$ & $\begin{array}{l}25.4 \pm \\
1.02 \mathrm{c}\end{array}$ & $\begin{array}{r}8.3 \pm \\
0.58 \mathrm{e}\end{array}$ & $\begin{array}{l}0.65 \pm \\
0.04 \mathrm{c}\end{array}$ & $\begin{array}{l}31.1 \pm \\
1.23 \mathrm{~d}\end{array}$ & $\begin{array}{l}25.9 \pm \\
1.02 \mathrm{c}\end{array}$ & $\begin{array}{c}8.8 \pm \\
0.58 \mathrm{~d}\end{array}$ & $\begin{array}{l}0.64 \pm \\
0.12 \mathrm{c}\end{array}$ \\
\hline $\begin{array}{l}\text { My }+2.5 \mathrm{mM} \\
\text { put }\end{array}$ & $\begin{array}{l}34.2 \pm \\
1.15 \mathrm{~b}\end{array}$ & $\begin{array}{l}24.5 \pm \\
1.13 \mathrm{c}\end{array}$ & $\begin{array}{c}9.8 \pm \\
0.68 \mathrm{~d}\end{array}$ & $\begin{array}{l}0.66 \pm \\
0.21 \mathrm{~d}\end{array}$ & $\begin{array}{l}34.6 \pm \\
1.10 \mathrm{c}\end{array}$ & $\begin{array}{l}26.2 \pm \\
1.13 \mathrm{c}\end{array}$ & $\begin{array}{l}10.8 \pm \\
0.68 \mathrm{c}\end{array}$ & $\begin{array}{l}0.63 \pm \\
0.21 \mathrm{c}\end{array}$ \\
\hline $\begin{array}{l}\text { My + } 5 \mathrm{mM} \\
\text { put }\end{array}$ & $\begin{array}{l}32.5 \pm \\
1.02 \mathrm{e}\end{array}$ & $\begin{array}{l}23.7 \pm \\
0.74 \mathrm{~d}\end{array}$ & $\begin{array}{c}8.4 \pm \\
0.67 \mathrm{~d}\end{array}$ & $\begin{array}{l}0.68 \pm \\
0.24 \mathrm{e}\end{array}$ & $\begin{array}{l}33.2 \pm \\
1.02 \mathrm{c}\end{array}$ & $\begin{array}{l}25.7 \pm \\
0.74 b\end{array}$ & $\begin{array}{r}9.0 \pm \\
0.67 \mathrm{c}\end{array}$ & $\begin{array}{l}0.67 \pm \\
0.20 \mathrm{e}\end{array}$ \\
\hline PGPR + My & $\begin{array}{l}33.4 \pm \\
1.01 \mathrm{c}\end{array}$ & $\begin{array}{l}25.5 \pm \\
1.11 \mathrm{c}\end{array}$ & $\begin{array}{l}10.9 \pm \\
0.68 \mathrm{c}\end{array}$ & $\begin{array}{l}0.66 \pm \\
0.18 \mathrm{c} \\
\end{array}$ & $\begin{array}{l}33.2 \pm \\
1.021 \mathrm{c}\end{array}$ & $\begin{array}{l}26.8 \pm \\
1.31 \mathrm{~b}\end{array}$ & $\begin{array}{l}11.1 \pm \\
0.68 \mathrm{~b}\end{array}$ & $\begin{array}{l}0.65 \pm \\
0.15 d\end{array}$ \\
\hline $\begin{array}{l}\text { PGPR+My+2.5 } \\
\text { mM put }\end{array}$ & $\begin{array}{l}36.5 \pm \\
1.23 \mathrm{a}\end{array}$ & $\begin{array}{l}29.8 \pm \\
1.30 \mathrm{a}\end{array}$ & $\begin{array}{l}13.8 \pm \\
0.68 \mathrm{a}\end{array}$ & $\begin{array}{l}0.58 \pm \\
0.06 \mathrm{a}\end{array}$ & $\begin{array}{l}36.9 \pm \\
1.13 \mathrm{a}\end{array}$ & $\begin{array}{l}31.8 \pm \\
1.30 \mathrm{a}\end{array}$ & $\begin{array}{l}14.2 \pm \\
0.68 \mathrm{a}\end{array}$ & $\begin{array}{l}0.57 \pm \\
0.03 \mathrm{a}\end{array}$ \\
\hline $\begin{array}{l}\text { PGPR+My+5m } \\
\text { Mput }\end{array}$ & $\begin{array}{l}33.9 \pm \\
1.32 \mathrm{~b}\end{array}$ & $\begin{array}{l}27.5 \pm \\
1.12 \mathrm{~b}\end{array}$ & $\begin{array}{l}10.5 \pm \\
1.03 \mathrm{c}\end{array}$ & $\begin{array}{l}059 \pm \\
0.03 \mathrm{a}\end{array}$ & $\begin{array}{l}33.7 \pm \\
1.42 \mathrm{c}\end{array}$ & $\begin{array}{l}27.5 \pm \\
1.12 \mathrm{~b}\end{array}$ & $\begin{array}{l}11.1 \pm \\
1.03 \mathrm{~b}\end{array}$ & $\begin{array}{l}059 \pm \\
0.05 \mathrm{~b}\end{array}$ \\
\hline
\end{tabular}

Mean values \pm SD followed by different letter are significantly different by ANOVA at 0.05 level.

Yield and its components as well as fruit quality

Table 7 shows that all tested treatments gave significant increase on total palm yield, fruit weight and flesh weight in both seasons compared with the control. Soil application of either PGPR or Mycorhiza, both at the rate of 85 $\mathrm{ml} /$ tree increased total yield/tree in the presence or absence of putrescine. Conjugation of both PGPR and Mycorhiza gave an additive effects in this respect. These data are being more evident when related to the control.

As for the effect of putrscine amine, the data demontested that it is more effective in increasing total yield/tree. Putrscine at the rate of $2.5 \mathrm{mM}$ was the best compared to the concentration of 0 (control) and $5 \mathrm{mM}$. The complemented treatments gave the highest values in this respect. 
Improving date palm yield and its components with putrescine application may be due to its effects on increasing photosynthates production and decreasing photosynthates consumption and injury to the membrane, thereby increasing crop productivity under reclaimed salinizied soils (salt stress). In this context, Tang and Newton (2005) attributed the positive effect of putrscine on increasing total yield under salinity to its effects on reducing salt induced oxidative damage by increasing the activities of antioxidant enzymes and decreasing lipid peroxidation, similarly to that found in the present investigation.

TABLE 7. Effects of plant growth promoting rhizobacteria (PGPR), Mycorhiza (My), Putresine amine alone or in combination on yield and its components of date palm (Phoenix dactylifera $\mathbf{L}$,) irrigated with saline water and grown in reclaimed saline soil during the two growing seasons (2013 and 2014).

\begin{tabular}{|c|c|c|c|c|c|c|}
\hline \multirow{3}{*}{ Treatments } & \multicolumn{6}{|c|}{ Growing seasons } \\
\hline & \multicolumn{3}{|c|}{ First season 2013} & \multicolumn{3}{|c|}{ Second season 2014} \\
\hline & $\begin{array}{c}\text { Yield/tree } \\
\text { (kg) }\end{array}$ & $\begin{array}{c}\text { Fresh Wt./ } \\
\text { fruit (g) }\end{array}$ & $\begin{array}{c}\text { Flesh Wt } \\
(\mathrm{g})\end{array}$ & $\begin{array}{c}\text { Yield/tree } \\
(\mathrm{kg})\end{array}$ & $\begin{array}{c}\text { Fresh Wt./ } \\
\text { fruit }(g)\end{array}$ & $\begin{array}{c}\text { Flesh Wt } \\
(\mathrm{g})\end{array}$ \\
\hline Control & $\begin{array}{l}126.1 \pm \\
2.01 \mathrm{~h}\end{array}$ & $\begin{array}{l}13.36 \pm \\
1.11 \mathrm{~d}\end{array}$ & $\begin{array}{l}10.31 \pm \\
1.17 \mathrm{e}\end{array}$ & $\begin{array}{c}123.7 \pm \\
1.11 \mathrm{j}\end{array}$ & $\begin{array}{l}13.64 \pm \\
1.24 \mathrm{c}\end{array}$ & $\begin{array}{l}10.45 \pm \\
1.42 \mathrm{~d}\end{array}$ \\
\hline $2.5 \mathrm{mM}$ put & $\begin{array}{l}150.4 \pm \\
2.71 \mathrm{c}\end{array}$ & $\begin{array}{l}15.72 \pm \\
1.24 \mathrm{~b}\end{array}$ & $\begin{array}{l}14.20 \pm \\
1.05 \mathrm{~b}\end{array}$ & $\begin{array}{l}154.4 \pm \\
2.42 \mathrm{c}\end{array}$ & $\begin{array}{l}15.95 \pm \\
1.35 \mathrm{~b}\end{array}$ & $\begin{array}{c}14.70 \pm 1.28 \\
b\end{array}$ \\
\hline $5 \mathrm{mM}$ put & $\begin{array}{l}130.1 \pm \\
2.05 \mathrm{~g}\end{array}$ & $\begin{array}{l}14.35 \pm \\
1.54 \mathrm{c}\end{array}$ & $\begin{array}{l}13.42 \pm \\
1.43 \mathrm{c}\end{array}$ & $\begin{array}{l}132.6 \pm \\
1.85 \mathrm{~h}\end{array}$ & $\begin{array}{l}14.95 \pm \\
1.14 \mathrm{c}\end{array}$ & $\begin{array}{l}13.85 \pm \\
1.30 \mathrm{c}\end{array}$ \\
\hline PGPR & $\begin{array}{c}135.6 \pm \\
1.85\end{array}$ & $\begin{array}{l}13.90 \pm \\
1.25 \mathrm{c}\end{array}$ & $\begin{array}{l}13.02 \pm \\
1.25 \mathrm{c}\end{array}$ & $\begin{array}{c}136.6 \pm \\
1.25 \mathrm{~g}\end{array}$ & $\begin{array}{l}13.67 \pm \\
1.25 \mathrm{c}\end{array}$ & $\begin{array}{l}12.44 \pm \\
1.15 \mathrm{c}\end{array}$ \\
\hline $\begin{array}{l}\text { PGPR+2.5mMp } \\
\text { ut }\end{array}$ & $\begin{array}{l}160.4 \pm \\
1.73 b\end{array}$ & $\begin{array}{l}16.45 \pm \\
1.43 b\end{array}$ & $\begin{array}{c}15.14 \pm \\
1.01 \mathrm{~b}\end{array}$ & $\begin{array}{l}164.4 \pm \\
1.70 \mathrm{~b}\end{array}$ & $\begin{array}{c}16.75 \pm \\
1.43 \mathrm{~b}\end{array}$ & $\begin{array}{c}15.36 \pm \\
1.14 \mathrm{~b}\end{array}$ \\
\hline \begin{tabular}{|l|}
$\begin{array}{l}\text { PGPR+ } 5 \mathrm{mM} \\
\text { put }\end{array}$ \\
\end{tabular} & $\begin{array}{l}140.1 \pm \\
1.75 \mathrm{e}\end{array}$ & $\begin{array}{l}14.26 \pm \\
1.25 \mathrm{~b}\end{array}$ & $\begin{array}{l}14.54 \pm \\
1.71 \mathrm{~b}\end{array}$ & $\begin{array}{l}143.4 \pm \\
1.45 \mathrm{f}\end{array}$ & $\begin{array}{l}14.46 \pm \\
1.25 \mathrm{c}\end{array}$ & $\begin{array}{l}14.85 \pm \\
1.21 \mathrm{~b}\end{array}$ \\
\hline My & $\begin{array}{l}126.7 \pm \\
1.75\end{array}$ & $\begin{array}{l}14.14 \pm \\
1.01 \mathrm{c}\end{array}$ & $\begin{array}{l}13.51 \pm \\
1.05 \mathrm{c}\end{array}$ & $\begin{array}{c}130.7 \pm \\
1.78 \mathrm{i}\end{array}$ & $\begin{array}{l}14.36 \pm \\
1.01 \mathrm{c}\end{array}$ & $\begin{array}{l}13.94 \pm \\
1.45 \mathrm{~b}\end{array}$ \\
\hline $\begin{array}{l}\text { My + } 2.5 \mathrm{mM} \\
\text { put }\end{array}$ & $\begin{array}{l}157.8 \pm \\
1.90 \mathrm{~d}\end{array}$ & $\begin{array}{l}15.14 \pm \\
1.71 \mathrm{~b}\end{array}$ & $\begin{array}{l}14.16 \pm \\
1.85 \mathrm{~b}\end{array}$ & $\begin{array}{l}159.2 \pm \\
1.65 \mathrm{e}\end{array}$ & $\begin{array}{l}15.75 \pm \\
1.71 \mathrm{~b}\end{array}$ & $\begin{array}{l}14.76 \pm \\
1.75 b\end{array}$ \\
\hline $\begin{array}{l}\text { My + 5 m } \\
\text { M put }\end{array}$ & $\begin{array}{l}135.1 \pm \\
2.04 \mathrm{f}\end{array}$ & $\begin{array}{l}14.32 \pm \\
1.05 \mathrm{c}\end{array}$ & $\begin{array}{c}13.24 \pm \\
1.33 \mathrm{c}\end{array}$ & $\begin{array}{l}137.1 \pm \\
1.25 \mathrm{~g}\end{array}$ & $\begin{array}{l}14.21 \pm \\
1.05 \mathrm{c}\end{array}$ & $\begin{array}{l}13.54 \pm \\
1.39 \mathrm{c}\end{array}$ \\
\hline PGPR + My & $\begin{array}{c}160.4 \pm \\
1.72 \mathrm{e}\end{array}$ & $\begin{array}{c}14.16 \pm \\
1.85 \mathrm{c}\end{array}$ & $\begin{array}{c}12.14 \pm 1.52 \\
\mathrm{c}\end{array}$ & $\begin{array}{c}163.4 \pm \\
1.05 \mathrm{e}\end{array}$ & $\begin{array}{c}14.48 \pm \\
1.85 \mathrm{c}\end{array}$ & $\begin{array}{c}12.34 \pm \\
1.42 \mathrm{c}\end{array}$ \\
\hline $\begin{array}{l}\text { PGPR+My + } \\
2.5 \text { m Mput }\end{array}$ & $\begin{array}{c}166.4 \pm \\
1.70 \mathrm{a}\end{array}$ & $\begin{array}{l}17.54 \pm \\
1.33 \mathrm{a}\end{array}$ & $\begin{array}{c}16.15 \pm \\
1.33 \mathrm{a}\end{array}$ & $\begin{array}{c}180.5 .4 \pm \\
1.58 \mathrm{a}\end{array}$ & $\begin{array}{l}18.12 \pm \\
1.55 \mathrm{a}\end{array}$ & $\begin{array}{c}16.45 \pm \\
1.63 \mathrm{a}\end{array}$ \\
\hline $\begin{array}{l}\text { PGPR+ My + } \\
5 \text { mM put }\end{array}$ & $\begin{array}{l}137.2 \pm \\
1.50 \mathrm{~d}\end{array}$ & $\begin{array}{l}16.14 \pm \\
1.52 b\end{array}$ & $\begin{array}{l}15.01 \pm \\
1.33 b\end{array}$ & $\begin{array}{l}139.6 \pm \\
1.30 \mathrm{~d}\end{array}$ & $\begin{array}{l}16.58 \pm \\
1.52 \mathrm{~b}\end{array}$ & $\begin{array}{l}15.34 \pm \\
1.75 b\end{array}$ \\
\hline
\end{tabular}

Mean values \pm SD followed by different letter are significantly different by ANOVA at 0.05 level.

Regarding fruit quality, data presented in Table 8 shows that application of PGPR, and/or My with or without Putresine to the date palm trees irrigated with salinized water and grown in the reclaimed saline soil increased concentrations of $\mathrm{N}, \mathrm{P}, \mathrm{K}$, crude protein (data not presented) and total carbohydrate in the date palm fruits. PGPR + My in the presence of Putresine at $2.5 \mathrm{mM}$ gave highest levels in this respect. The percentage of vitamin $\mathrm{C}$, total soluble solids in date palm fruits were also increased due to all treatments used in the two growing

Egypt. J. Hort. Vol. 42, No. 1 (2015) 
seasons. Although recovery of fruit production and their quality was observed from all three exogenously addition of PGPR, My and/or put., put is the most detective treatments. Among different concentration of put, $2.5 \mathrm{mM}$ resulted highest increase in date fruits productivity and their quality compared to other treatments. The additive effect of put was more pronounced incombination with PGPR+My. The highest values of Vit C and TSS were existed with PGPR, Mycorhiza and Putresine at $2.5 \mathrm{mM}$. These data are being important from the view of fruit quality since, that could prolong the shelf time. Therefore, put would be the best candidate for not only recovering productivity of date palm but also improving fruit quality. In this respect, Mohamed and Tarpley (2009) found that abiotic stress can decrease crop yields by decreasing crop growth duration, suppressing floral bud development and decreasing pollen viability. The same authors (2011) attributed the suppressing of crop yield under stress condition to a shortage of photosynthetic assimilates supplied to the floral buds and/or inability of floral buds to mobilize carbohydrates. Increased respiration and decreased photosynthesis and stability were also recorded (Freire et al., 2009)

TABLE 8. Effects of plant growth promoting rhizobacteria (PGPR), Mycorhiza (My), Putresine amine alone or in combination on fruit quality of date palm (Phoenix dactylifera $\mathrm{L}$,) zaghloul irrigated with saline water and grown in reclaimed saline soil (Means of the two growing seasons 2013 and 2014).

\begin{tabular}{|c|c|c|c|c|c|c|c|}
\hline \multirow[b]{2}{*}{ Treatments } & \multirow{2}{*}{$\begin{array}{c}\text { Total } \\
\text { sugars } \\
\text { mg/g } \\
\text { F.Wt. }\end{array}$} & \multirow{2}{*}{$\begin{array}{c}\text { Total } \\
\text { Carbohy- } \\
\text { drate } \\
\%\end{array}$} & \multirow{2}{*}{$\begin{array}{c}\text { Total } \\
\text { Soluble } \\
\text { Solids } \\
\text { \%TSS }\end{array}$} & \multirow{2}{*}{$\begin{array}{l}\text { Vit. C } \\
\text { mg/100g } \\
\text { F.Wt. }\end{array}$} & \multicolumn{3}{|c|}{ Mg/g D. Wt. } \\
\hline & & & & & $\mathbf{N}$ & $\mathbf{P}$ & $\mathbf{K}$ \\
\hline Control & $\begin{array}{l}18.8 \pm \\
1.56 \mathrm{~d}\end{array}$ & $\begin{array}{l}53 . .7 \pm \\
1.23 \mathrm{e}\end{array}$ & $\begin{array}{c}23.11 \pm \\
0.32 \mathrm{~d}\end{array}$ & $\begin{array}{l}43 . .6 \pm \\
1.01 \mathrm{i}\end{array}$ & $\begin{array}{l}10.6 \pm \\
1.05 \mathrm{e}\end{array}$ & $\begin{array}{c}2.2 \pm \\
0.37 \mathrm{~d}\end{array}$ & $\begin{array}{c}20.02 \pm \\
1.21 \mathrm{~d}\end{array}$ \\
\hline $2.5 \mathrm{mM}$ put & $\begin{array}{l}23.3 \pm \\
1.33 \mathrm{~b}\end{array}$ & $\begin{array}{l}60.9 \pm \\
1.26 \mathrm{c}\end{array}$ & $\begin{array}{c}24.03 \pm 0 . \\
41 \mathrm{c}\end{array}$ & $\begin{array}{l}67.9 \pm \\
1.21 \mathrm{c}\end{array}$ & $\begin{array}{l}14.4 \pm \\
1.24 \mathrm{c}\end{array}$ & $\begin{array}{c}3.8 \pm \\
0.74 b\end{array}$ & $\begin{array}{l}22.9 \pm \\
1.23 \mathrm{~b}\end{array}$ \\
\hline $5 \mathrm{mM}$ put & $\begin{array}{l}22.2 \pm \\
1.13 \mathrm{~b}\end{array}$ & $\begin{array}{l}57.3 \pm \\
1.15 \mathrm{~d}\end{array}$ & $\begin{array}{c}23.78 \pm \\
0.35 \mathrm{c}\end{array}$ & $\begin{array}{l}54.3 \pm \\
1.13 \mathrm{~g}\end{array}$ & $\begin{array}{l}13.1 \pm \\
1.11 \mathrm{c}\end{array}$ & $\begin{array}{r}3.5 \pm \\
0.75 b\end{array}$ & $\begin{array}{l}21.3 \pm \\
1.15 \mathrm{c}\end{array}$ \\
\hline PGPR & $\begin{array}{l}21.7 \pm \\
1.11 \mathrm{c}\end{array}$ & $\begin{array}{l}56.8 \pm \\
1.14 \mathrm{~d}\end{array}$ & $\begin{array}{c}23.87 \pm \\
0.35 \mathrm{c}\end{array}$ & $\begin{array}{l}57.8 \pm \\
1.16 \mathrm{f}\end{array}$ & $\begin{array}{l}12.6 \pm \\
1.32 \mathrm{c}\end{array}$ & $\begin{array}{c}3.0 \pm \\
0.45 b\end{array}$ & $\begin{array}{l}20.8 \pm \\
1.14 \mathrm{c}\end{array}$ \\
\hline $\begin{array}{l}\text { PGPR+2mM } \\
\text { put }\end{array}$ & $\begin{array}{l}25.1 \pm \\
1.43 \mathrm{a}\end{array}$ & $\begin{array}{l}63.8 \pm \\
1.02 \mathrm{~b}\end{array}$ & $\begin{array}{c}24.18 \pm \\
0.35 \mathrm{~b}\end{array}$ & $\begin{array}{l}71.8 \pm \\
1.09 \mathrm{~b}\end{array}$ & $\begin{array}{l}17.3 \pm \\
1.27 \mathrm{~b}\end{array}$ & $\begin{array}{c}4.2 \pm \\
0.75 \mathrm{a}\end{array}$ & $\begin{array}{l}23.1 \pm \\
1.02 b\end{array}$ \\
\hline $\begin{array}{l}\text { PGPR+5mM } \\
\text { put }\end{array}$ & $\begin{array}{l}23.5 \pm \\
1.24 \mathrm{~b}\end{array}$ & $\begin{array}{l}58.2 \pm \\
1.24 \mathrm{c}\end{array}$ & $\begin{array}{c}23.94 \pm \\
0.26 \mathrm{c}\end{array}$ & $\begin{array}{l}59.2 \pm \\
1.20 \mathrm{e}\end{array}$ & $\begin{array}{l}14.6 \pm \\
1.15 \mathrm{c}\end{array}$ & $\begin{array}{r}3.7 \pm \\
0.55 \mathrm{a}\end{array}$ & $\begin{array}{l}22.2 \pm \\
1.24 b\end{array}$ \\
\hline My & $\begin{array}{l}21.7 \pm \\
1.20 \mathrm{c}\end{array}$ & $\begin{array}{c}54.4 \pm \\
1.22\end{array}$ & $\begin{array}{c}23.76 \pm \\
0.32 \mathrm{c}\end{array}$ & $\begin{array}{l}61.4 \pm \\
1.27 \mathrm{e}\end{array}$ & $\begin{array}{l}12.2 \pm \\
1.28 \mathrm{~d}\end{array}$ & $\begin{array}{c}2.9 \pm \\
0.42 \mathrm{c}\end{array}$ & $\begin{array}{l}21.4 \pm \\
1.22 \mathrm{c}\end{array}$ \\
\hline $\begin{array}{l}\text { My }+2.5 \mathrm{mM} \\
\text { put }\end{array}$ & $\begin{array}{l}24.1 \pm \\
1.20 \mathrm{a} \\
\end{array}$ & $\begin{array}{l}59.3 \pm \\
1.13 \mathrm{c} \\
\end{array}$ & $\begin{array}{c}24.16 \pm \\
0.31 b\end{array}$ & $\begin{array}{l}62.3 \pm \\
1.11 \mathrm{~d} \\
\end{array}$ & $\begin{array}{l}15.2 \pm \\
1.35 \mathrm{~b}\end{array}$ & $\begin{array}{r}3.6 \pm \\
0.58 \mathrm{~b}\end{array}$ & $\begin{array}{l}22.3 \pm \\
1.13 b\end{array}$ \\
\hline $\mathrm{My}+5 \mathrm{mM}$ pu & $\begin{array}{l}23.4 \pm \\
1.45 b\end{array}$ & $\begin{array}{l}57.1 \pm \\
1.16 \mathrm{~d}\end{array}$ & $\begin{array}{c}23.54 \pm \\
0.25 \mathrm{c}\end{array}$ & $\begin{array}{l}60.1 \pm \\
1.13 \mathrm{e}\end{array}$ & $\begin{array}{l}12.6 \pm \\
1.02 \mathrm{~d}\end{array}$ & $\begin{array}{c}3.0 \pm \\
0.45 b\end{array}$ & $\begin{array}{l}22.1 \pm \\
1.16 b\end{array}$ \\
\hline PGPR + My & $\begin{array}{l}22.7 \pm \\
1.21 \mathrm{~b}\end{array}$ & $\begin{array}{l}58.7 \pm \\
1.30 \mathrm{c}\end{array}$ & $\begin{array}{c}23.72 \pm \\
0.25 \mathrm{c}\end{array}$ & $\begin{array}{l}63.7 \pm \\
1.34 \mathrm{c}\end{array}$ & $\begin{array}{l}13.1 \pm \\
1.17 \mathrm{c}\end{array}$ & $\begin{array}{c}3.7 \pm \\
1.05 \mathrm{a}\end{array}$ & $\begin{array}{l}21.7 \pm \\
1.34 \mathrm{c}\end{array}$ \\
\hline $\begin{array}{l}\text { PGPR+My+ } \\
2.5 \mathrm{mMput}\end{array}$ & $\begin{array}{l}25.9 \pm \\
1.21 \mathrm{a}\end{array}$ & $\begin{array}{l}66.8 \pm \\
1.18 \mathrm{a}\end{array}$ & $\begin{array}{c}24.83 \pm \\
0.32 \mathrm{a}\end{array}$ & $\begin{array}{l}76.8 \pm \\
1.15 \mathrm{a}\end{array}$ & $\begin{array}{l}19.8 \pm \\
1.23 \mathrm{a}\end{array}$ & $\begin{array}{c}4.6 \pm \\
0.75 \mathrm{a}\end{array}$ & $\begin{array}{l}24.8 \pm \\
1.15 \mathrm{a}\end{array}$ \\
\hline $\begin{array}{l}\text { PGPR+My+5 } \\
\text { mM put }\end{array}$ & $\begin{array}{l}23.8 \pm \\
1.61 \mathrm{~b}\end{array}$ & $\begin{array}{l}60.2 \pm \\
1.05 \mathrm{c}\end{array}$ & $\begin{array}{c}23.31 \pm \\
0.28 \mathrm{c}\end{array}$ & $\begin{array}{l}63.9 \pm \\
1.15 \mathrm{c}\end{array}$ & $\begin{array}{l}15.3 \pm \\
1.27 \mathrm{~b}\end{array}$ & $\begin{array}{r}4.0 \pm \\
0.68 \mathrm{a}\end{array}$ & $\begin{array}{l}22.9 \pm \\
1.15 b\end{array}$ \\
\hline
\end{tabular}

Mean values \pm SD followed by different letter are significantly different by ANOVA at 0.05 level. 
The important role of PGPR and My on salinized soil may exhibit through increment in nutrient elements availability by reducing soil $\mathrm{pH}$, increasing the exchangeable capacity and reducing their losses by leaching as well as the ability of organic chelating agents to protect the nutrient elements against the conversion to unavailable forms (Abada et al., 2010 and Kassem, 2012). Most plants adapted to dry stress environments have mycorrhizal symbiosis, which improves water and nutrient supply but is also a sink for carbohy drates and many consume 5-10\% of total photosynthate (Koch \& Johnson, 1984 and Fogel, 1985). Results of Kumar et al. (2014) indicated that the nutritional status might be attribute to enhance inorganic and organic nutrient absorption by biofertilizers which in turn make the essential nutrient available to the promoting growth and increase nutrient content in leaves. The factor leads to increase photosynthetic surface area there by indicating the sufficient utilization of solar radiation ultimately leading to production of assimilates.

It could be concluded that putresine, some microbial species strains could play an important role for explaining how date palm can be tolerant and adapt to stress condition. The selected plant growth promoting rhizobbacteria and mycrorhiza in the presence of Putrescine enable date palm to increase their tolerance and adaptation to reclaimed-salinizied areas as one of many abiotic stresses. Moreover, the interaction treatments between PGPR and Putresine under salt and osmotic stress condition could affect not only the productivity of date palm but also the properties of soil. Further investigation are needed to explain the mechanisms those develop in date palm under stress condition. Thereby, selection of certain microorganisms from stressed ecosystems would insert in to the concept of biotechnology application in agriculture mangements. The physiological behavior mechanism of the plants under these conditions is also needed in order to understand the role of Put in reducing salt-stress induced oxidative.

\section{References}

A.O.A.C. (1995) Association of Official Agricultural Chemists, "Official Methods of Analysis", $15^{\text {th }}$ ed., A.O.A.C., Washington, DC.

Abada, M.A.M., Asmaa Ibrahim, A. and Sawsan Bondok, A. (2010) How to reduce problems of soil and irrigation water salinity in superior vineyards? Minufiya. J. Agric. Res., 35, 1477-1497.

Ahmed,M. and kibret, M. (2014) mechanisms and application of plant growth promoting rhizobacteria: current perspective, J. king Saud Univ. Sci., 26, 1-20.

Amberger, A. (1954) Einflu, von kalium und stickstoff auf ferment and kolenhydrathaus halt von grunland pflanzen. J. Plant Nutr. Soil Sci., 66, 211-222.

Anonymous (2010) http://faostat.fao.org/site/567/Deskt 
Aribaud, M., Carrè, M. and Martin-Tanguy, I. (1994) Poly amine metabolism and in vitro cell multiplication and differentiation in leaf explants of chrysanthemum morifolium. Ramat. Plant Growth Regul. 15, 143-155.

Beauchamp, C. and Fridovich, I. (1971) Superoxide dismutase: Improved assays and an assay applicable to acry lamide gels. Anal. Biochem., 44, 276-287.

Bhatti K.H., Sehrish Aikhalid, N. and Khalid, H. (2013) Effect of exogenous application of glycine betaine on wheat (Triticum aestivcum, L.) under heavy metal stress. Middle-East J.of Sires., 14 (1), 130-137.

Black, C.A., Evans, D.O., Ensminger, L.E., White, J.L., Clark, F.E. and Dinauer, R.C. (1982) "Methods of Soil Analysis" Part.2 Chemical and Microbiological Properties, $2^{\text {nd }}$ ed., Soil Sci., of Am. Inch. Publ., Madison, Wisconsin, USA.

Borsani, O., Valpuesta, V. and Botella M.A. (2001) Evidence for a role of salicy lic acid in the oxidative damage generated by $\mathrm{NaCl}$ and osmotic stress in Arabidopsis seedlings. Plant Physiol., 126,1024-1030.

Bremner, J.M. and Muluaney, C.S. (1982) Total nitrogen in : Method of Soil Analysis part2. A.L.R.H. Miller and D.R. keeney (Ed.). Amer. Soc. Agron. Madison. W.I. USA. pp. 595-624.

Chapman, H.D. and P.E. Pratt (1982) "Method of Analysis of Soil, Plant and Water" $2^{\text {nd }}$ ed., California Univ., Agric. Division. p. 170.

Cha-um, S., Supaibulwatana, K. and Kiodmanee, C. (2006) Water reaction, photosynthetic ability and growth of Thai Jasmine rice (Oryza sativa, L. ssp. Indic acv. KDML105) to salt stress by application of exogenous glycine betaine and choline. J. Agroon. And crop. Sc 19225-36.

Cowley, T. and Walters, D.R. (2002) Polyamine metabolism in barley reacting hypersensitively to the powdery mildew fungus Blumeria graminis f.sp. Hordei. Plant Cell Environ., 25, 461-468.

Dhindsa, R. S., Plumb-Dhindsa, P. and Throne, T.A. (1981) Leaf senescence: occurrelated with increased leaves of membrane permeability and lipid peroxidation and decreased levels of superoxide dismutase and catalase. J. Exp. Bot., 32, 93-101.

Doberiner, J. (1978) Influence of environmental factors on the occurrence of S., lipoferum in soil and roots. Ecol. Bull., (Stockholm) 26, 343-352.

Dong, S., Cheng, L., Scagel, C.F. and Fuchigami, L.H. (2005) Timing of urea application affects leaf and root $\mathrm{N}$ uptake in young Fuji/M9 apple trees. J. Hortic. Sci. Biotech., 80, 116-120.

Dowson, (1957) "Plant Diseases Due to Bacteria", $2^{\text {nd }}$ ed., Cambridge University Press, pp. 169-177.

Dubey, R.S. and Rani, M. (1989a) influence of $\mathrm{NaCl}$ salinity on growth and metabolic status of protein and amino acids in rice seedlings. J. Agron. Crop Sci., 162, 97-106. 
Dubey, R.S. and Rani, M. (1989b) salinity induces accumulation of free amino acids in germinating rice seeds differing in salt tolerance. J. Agron. Crop Sci., 163, 236-247.

El-Khawaga, A.S. (2013) Effect of anti-salinity agents on growth and fruiting of different date palm cultivars. Asian Journal of Crop Science 5(1): 65-80.

Faive - Rampant, O., Kevers C., Dommes J. and Gasper T. (2000) The recalcitrance to rooting of the micropropagated shoot of the race tobacco mutant: Implications of poly amines and of the poly amine metabolism. Plant Physiol. Biochem., 38, 441-448

Fogel, R. (1985) Roots as primary producers in blow-ground ecosystem. In: Ecological Interactions in Soil, A.H. Fitter, D. Atkinson, D.J. Read and M.B. Usher, (Ed.). Oxford UK: Blackwell.

Foyer, C.H. and Halliwell, B. (1976) Presenence of glutathione and glutathione reductase in chloroplasts: a proposed tole in ascorbic acid metabolism. Planta, 133, 21-25.

Freire, E. de A., Laime, E.M.M., Navilta, V. do N., Lima, V.L. de and Santos, J.Z. dos. (2009) Análise dos riscos de salinidade do solo do perímetro irrigado de Forquilha, Ceará. Revista Educação Agrícola Superior, 24 (2), 62-66

Gerdemann, J.W. and Nicolson, T.H. (1963) Spores of my corrhizal Endogone extracted from soil by wet sieving and decanting. Trans. Br. Mycol. Soc., 46, 235-244.

Ghoulam, C. and Fares, K. (2001) Effect of salinity on seed germination and early seedling growth of sugar beet (Beta vulgris L.) Sedci.an Techol., 29, 357-64.

Groppa, M.D., Tomaro, M.L. and Benvids, M.P., (2001). Polyamines as protectors against cadmium or copper- induced oxidative damage in sunflower leaf discs. Plant Sci. 161: 481-488.

Groppa, M.D., Benavides, M.P and Tomaaro, M.L. (2003) Poly amine metabolism in sunflower and wheat leaf disease under cadmium or copper stress. Plant Sci., 164, 293-299

Heidari, M. and Golpayegani,A.A. (2012) Effect of water stress and inoculation with plant growth promoting rhizobacteria (PGPR) on antioxidant status and photosynthetic pigments in basil (Ocimum basilicum, L.). J. Sandi Soc. Agric. Sci., 11(1), 57-61.

Helaly, M.N.M. and Hanan El-Hosieny A.R. (2011) Combined effects between genotypes and salinity on sweet orange during the developmental stages of its micropropagation. Research Journal of Botany, 6 (2), 38-57.

Jakson, M.L. (1973) Soil chemical analy sis prentice-Hall of India. Private new Delhi.

Kassem, H.A. (2012) The response of date palm to calcareous soil fertilization. J. Soil Sci. Plant Nut., 12, 45-58.

Koch K. and Johnson, C.R. (1984) Photosynthetic partitioning in split-rot citrus seedlings with mycorrhizal and nonmicorrizal root system. Plant Physiol., 75, 26.

Egypt. J. Hort. Vol. 42, No. 1 (2015) 
Koshioka, M., Harada G., Noma, M., Sassa, T., Ogiama, K., Taylor, J.S., Rood, S.B., Legge R.L. and Phris, R.P. (1983) Reversed phase C18 high performance liquid Chromatography of acidic and conjugated gibberellins. J. Chromatogr, 256, 101-115.

Kumar, A., Ram, R. B., Meena, M.L., Raj, U. and Anand, A.K. (2014) Effect of Bio fertilizers on nutritional characteristics in aonla seedling and grafted plants. International J. of Science and Nature, 5 (2), 258 -260.

Larkindale, J., Hall, J.D., Knight, M.R. and Vierling, E. (2005) Heat stress phenoty pes of arabidopsis mutants implicate multiple signaling pathways in the acquisition of thermotolerance. Plant Physiol., 138 (2), 882-897.

Liu, X. and Huang, B. (2000) Carbohydrate accumulation in relation to heat stress tolerance in two creeping bentgrass cultivars. J. Am. Soc. Hort. Sc., 125, 442-447.

Makela, P., Karkkasuen, J. and Someraslo, S. (2000) Effect of glycine betaine on chloroplast ultrastracture chlorophyll and protein content and RUBISCO activations in tomato grown under drought and salinity. Biol. Plant., 3, 471-475.

Minura, T., Kura-Hotta, M., Tsujimura, T., Ohnishi, M., Miura, M., Okazaki, Y., Mimura, M., Maeshima, M. and Washitani-Nemoto, S. (2003) Rapid increase of vacuolar. Volume in response to salt stress. Planta, 216, 397-402.

Mohammadi, K. and Sohrabi, Y. (2012) Becterial biofertilizers for sustainable crop ARPN Journal of Agricultural and Biological Science. 7 (5), 307-316.

Mohammed, A.R. and Tarpley, L. (2011) Characterization of rice (Oryza sativa,L) physiological response to a-tocopherol, gly cine betaine or salicy lic acid application. $J$. Agric Sc., 3, 3-13.

Mohommed, A.R. and Tarpley, L. (2009) Impact of high night time temperature on respiration, membrane stability, antioxidant capacity and yield of rice plants. Crop Sci., 49, 313-322.

Nag, S., Saha, K. and Choudhuri, M.A. (2000) A rapid and sensitive assay method for measuring amino oxidase based on hydrogen peroxide-titanium complex formation. Plant Sci., 157, 157-163.

Nakano, Y. and Asada, K. (1981) Hydrogen peroxide is scavenged by ascorbate specific peroxidase in spinach chloroplast. Plant Cell Physiol., 22, 867-880.

Nicander, B., Stahi, U., Bjorkman, P.O. and Tillberg, E. (1993) Immunoaffinity coPurification purification of cytokinins and analysis by high-performance liquid chromatography with ultraviolet spectrum-detection. Planta, 189, 312-320.

Olsen, S.R. and Sommers, L.E. ( 1982) Phosphorus. In: Methods of Soil Analysis, Part 2, Page, A.L., Keeney, D.R. (Ed.), pp. 403-430 . Amer. Soc. Agron. No. 9, Madison. WI, USA.

Rasmia, S.S. Darwesh' (2013) Improving growth of date palm plantlets grown under salt, Annals of Agricultural Science, $\mathbf{5 8}$ (2), 247-256. 
Sadasivam, S. and Manickam, A. (1996) "Biochemical Methods", New Age International Publishers, New Delhi, pp. 1-255.

Salama, A.S.M., Omima M. El-Sayed and El-Gammal, O.H.M. (2014) Effect of effective microorganisms(EM) and potassium sulphate on productivity and fruit quality of "Hayany" date palm grown under Salinity Stress. Journal of Agriculture and Veterinary Science, 7, 90-99. www.iosrjournals.org

SAS (2003) SAS/STARTR User's Guide: Statistics. Ver. 9.1, SAS Institute Inc., Cary, NC, USA.

Seckin, B., Turkan, I., Sekmen, A.H. and Ozfidan, C. (2010) The role of antioxidant defense systems at differential salt tolerance of Hordeum marinum Huds. (sea barleygrass) and Hordeum vulgare L. (cultivated barley). Environ. Exp. Bot., 69, 7685.

Snedecor, G.W. and Cochran, W.G. (1980) "Statistical Methods", $7^{\text {th }}$ ed., The Iowa State Univ. Press, Iowa, U.S.A.,

Tang, W. and Newton, R.J. (2005) Polyamines reduced salt-induced oxidative damage by increasing the activities of antioxidant enzymes and decreasing lipid peroxidation in Virginia pine. Plant Growth Reg., 46, 31-43.

Wahid, A. and Shabbir, A. (2005) Induction of heat stress tolerance in barley seedlings by pre-sowing seed treatment with gly cine betaine. Plant growth Reg., 46, 133-141.

Wettestein, D. (1957) Chlorophyll-lethal under submicroscopic from wech selder plastiden. Expt. Cell Res., 12, 427-500.

Zapata, L., Leonor Pena-Chocarro, Guillem Perez-Jord ' a,' and Hans-Peter S tika (2004) Effect of anti-salinity agents on growth and fruiting of different date palm cultivars. Journal of World Prehistory, 18 (4), 283-225.

Zhu, J.K. (2002) Salt and drought stress signal transduction in plants. Ann. Rev. Plant Biol., 53, 247-273.

Zhu, J.K., Chinnusamy, V. and Jagendorf, A. (2005) Understanding and improving salt tolerance in plants. Crop. Sci., 45, 437- 448.

(Received 8/3/2015;

accepted 26/5/2015) 


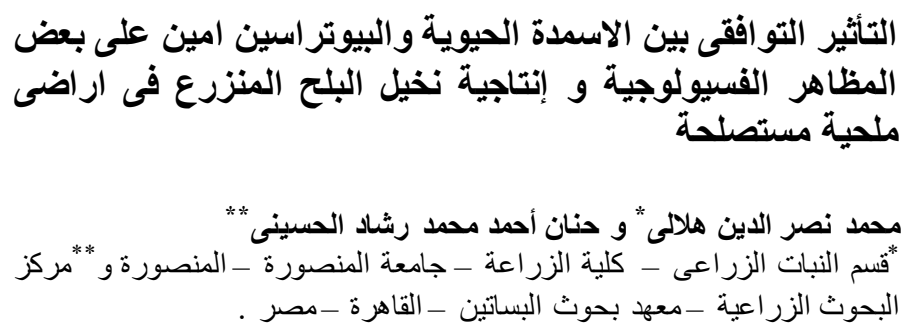

تلعب مركبات الأمين دورا هاما في استجابة النبات للظروف البيئية الضارة بما

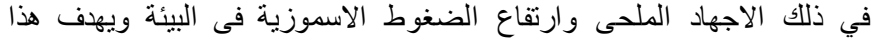

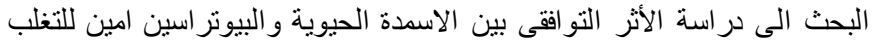

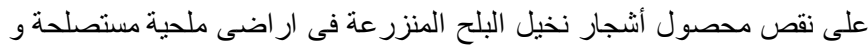

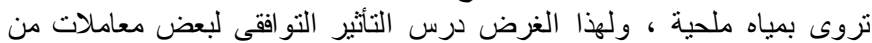

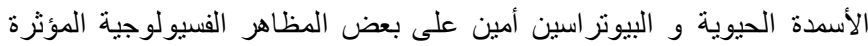

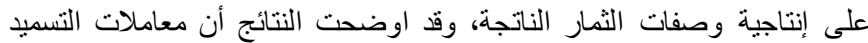

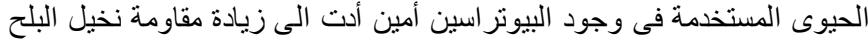

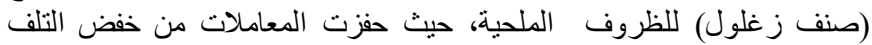

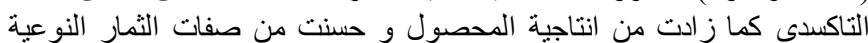

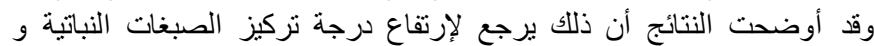

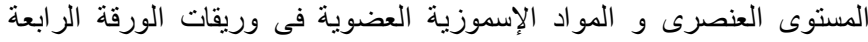
التى تم تحليلها فسيولوجيا إضافة إلى زيادة درجة تركيز المنشطات الإنية الهرمونية

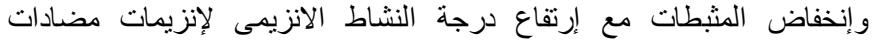

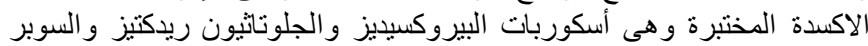

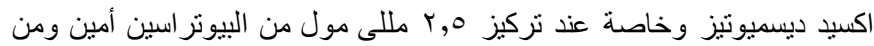

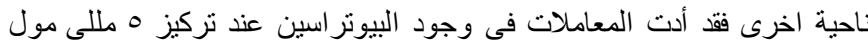

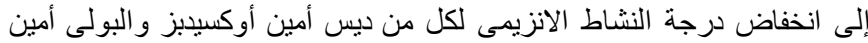

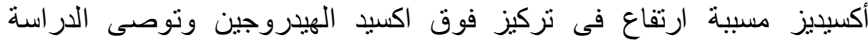

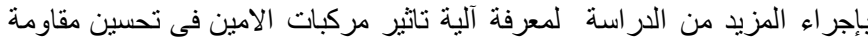
الظروف البيئية الغير ملائمة. 